\title{
MULTIFRACTALITY OF PRODUCTS OF GEOMETRIC ORNSTEIN-UHLENBECK-TYPE PROCESSES
}

\author{
V. V. ANH, ${ }^{*}$ Queensland University of Technology \\ NIKOLAI N. LEONENKO, ${ }^{* *}$ Cardiff University \\ NARN-RUEIH SHIEH, ${ }^{* * *}$ National Taiwan University
}

\begin{abstract}
We investigate the properties of multifractal products of geometric Ornstein-Uhlenbeck (OU) processes driven by Lévy motion. The conditions on the mean, variance, and covariance functions of the resulting cumulative processes are interpreted in terms of the moment generating functions. We consider five cases of infinitely divisible distributions for the background driving Lévy processes, namely, the gamma and variance gamma distributions, the inverse Gaussian and normal inverse Gaussian distributions, and the $z$-distributions. We establish the corresponding scenarios for the limiting processes, including their Rényi functions and dependence structure.
\end{abstract}

Keywords: Multifractal products; long-range dependence; geometric Ornstein-Uhlenbeck process; Lévy process; infinitely divisible distribution

2000 Mathematics Subject Classification: Primary 60G57; 60G10; 60G17

\section{Introduction}

Multifractal models have been used in many applications in hydrodynamic turbulence, finance, genomics, computer network traffic, etc. (see Kolmogorov (1941), (1962), Kahane (1985), (1987), Gupta and Waymire (1993), Novikov (1994), Frisch (1995), Mandelbrot (1997), and Falconer (1997)). Harte (2001) and Riedi (2003) contain an extensive bibliography of the subject. There are many ways to construct random multifractal measures such as via the binomial cascade and branching processes (see Kahane (1985), (1987), Gupta and Waymire (1993), Molchan (1996), Falconer (1997), Barral and Mandelbrot (2002), Riedi (2003), Mörters and Shieh (2002), (2004), (2008), and Shieh and Taylor (2002)). Most of these multifractal models are not designed to cover other important features such as tractable dependence structure or a natural form of the singularity spectrum (see, for example, Novikov (1994) and Riedi (2003)). Jaffard (1999) showed that Lévy processes (except Brownian motion and the Poisson process) are multifractal; but, since the increments of a Lévy process are independent, this class excludes the effects of tractable dependence structures. Moreover, Lévy processes have a linear singularity spectrum while real data often exhibit a strictly concave spectrum.

This paper follows a different approach. We consider multifractal products of stochastic processes as defined in Kahane (1985), (1987) and Mannersalo et al. (2002), but we provide

\footnotetext{
Received 31 May 2007; revision received 16 October 2008.

* Postal address: School of Mathematical Sciences, Queensland University of Technology, GPO Box 2434, Brisbane, QLD 4001, Australia. Email address: v.anh@qut.edu.au

** Postal address: Cardiff School of Mathematics, Cardiff University, Senghennydd Road, Cardiff CF24 4YH, UK.

Email address: leonenkon@ cardiff.ac.uk

*** Postal address: Department of Mathematics, National Taiwan University, Taipei 10617, Taiwan.

Email address: shiehnr@math.ntu.edu.tw
} 
a new interpretation of the conditions on the mean, variance, and covariance functions of the resulting cumulative processes in terms of the moment generating functions. This approach is more useful for our development. We show that the logarithms of the corresponding limiting processes have an infinitely divisible distribution such as the gamma and variance gamma distributions (resulting in the log-gamma and log-variance gamma scenarios, respectively), and the inverse Gaussian and normal inverse Gaussian distributions (yielding the log-inverse Gaussian and log-normal inverse Gaussian scenarios, respectively). We describe the behaviour of their $q$ th-order moments and Rényi functions, which are nonlinear, hence displaying their multifractality. A property on the dependence structure of the limiting processes, leading to their possible long-range dependence, is also obtained. Our exposition relies on some results of Mannersalo et al. (2002) on the basic properties of multifractal products of stochastic processes. We should also note some related results in Barndorff-Nielsen and Schmiegel (2004), who introduced some Lévy-based spatiotemporal models for parametric modelling of turbulence. Log-infinitely divisible scenarios related to independently scattered random measures were investigated in Schmitt (2003), Schmitt and Marsan (2001), and Bacry and Muzy (2003).

\section{Multifractal products of stochastic processes}

This section recaptures some basic results on multifractal products of stochastic processes as developed in Kahane (1985), (1987) and Mannersalo et al. (2002). We provide a new interpretation of their conditions based on the moment generating functions, which is useful for our exposition.

We introduce the following conditions.

(C1) Let $\Lambda(t), t \in \mathbb{R}_{+}=[0, \infty)$, be a measurable, separable, strictly stationary, positive stochastic process with $\mathrm{E} \Lambda(t)=1$.

We call this process the mother process and consider the following setting.

(C2) Let $\Lambda^{(i)}, i=0,1, \ldots$, be independent copies of the mother process $\Lambda$, and let $\Lambda_{b}^{(i)}$ be the rescaled version of $\Lambda^{(i)}$ :

$$
\Lambda_{b}^{(i)}(t) \stackrel{\mathrm{D}}{=} \Lambda^{(i)}\left(t b^{i}\right), \quad t \in \mathbb{R}_{+}, i=0,1,2, \ldots,
$$

where the scaling parameter $b>1$ and $\stackrel{\text { D, }}{=}$ denotes equality in finite-dimensional distributions.

Moreover, in the examples of Section 4, the stationary mother process satisfies the following condition.

(C3) For $t \in \mathbb{R}_{+}$, let $\Lambda(t)=\mathrm{e}^{X(t)}$, where $X(t)$ is a stationary process with $\mathrm{E} X^{2}(t)<\infty$.

We denote by $\boldsymbol{\theta} \in \Theta \subseteq \mathbb{R}^{p}, p \geq 1$, the parameter vector of the distribution of the process $X(t)$, and assume that there exist a marginal probability density function $p_{\theta}(x)$ and a bivariate probability density function $p_{\theta}\left(x_{1}, x_{2} ; t_{1}-t_{2}\right)$ such that the moment generating function

$$
M(\zeta)=\mathrm{Ee}^{\zeta X(t)}
$$

and the bivariate moment generating function

$$
M\left(\zeta_{1}, \zeta_{2} ; t_{1}-t_{2}\right)=\operatorname{E} \exp \left\{\zeta_{1} X\left(t_{1}\right)+\zeta_{2} X\left(t_{2}\right)\right\}
$$

exist. 
Conditions (C1)-(C3) yield

$$
\begin{gathered}
\operatorname{E~} \Lambda_{b}^{(i)}(t)=M(1)=1, \\
\operatorname{var} \Lambda_{b}^{(i)}(t)=M(2)-1=\sigma_{\Lambda}^{2}<\infty, \quad \sigma_{\Lambda}^{2}=\text { constant } \\
\operatorname{cov}\left(\Lambda_{b}^{(i)}\left(t_{1}\right), \Lambda_{b}^{(i)}\left(t_{2}\right)\right)=M\left(1,1 ;\left(t_{1}-t_{2}\right) b^{i}\right)-1, \quad b>1 .
\end{gathered}
$$

We define the finite product processes by

$$
\Lambda_{n}(t)=\prod_{i=0}^{n} \Lambda_{b}^{(i)}(t)=\exp \left\{\sum_{i=0}^{n} X^{(i)}\left(t b^{i}\right)\right\}
$$

and the cumulative processes by

$$
A_{n}(t)=\int_{0}^{t} \Lambda_{n}(s) \mathrm{d} s, \quad n=0,1,2, \ldots,
$$

where the $X^{(i)}(t), i=0, \ldots, n$, are independent copies of a stationary process $X(t), t \geq 0$. $\mathbb{R}_{+}$:

We also consider the corresponding positive random measures defined on Borel sets $B$ of

$$
\mu_{n}(B)=\int_{B} \Lambda_{n}(s) \mathrm{d} s, \quad n=0,1,2, \ldots
$$

Kahane (1987) proved that the sequence of random measures $\mu_{n}$ converges weakly almost surely to a random measure $\mu$. Moreover, given a finite or countable family of Borel sets $B_{j}$ on $\mathbb{R}_{+}$, it holds that $\lim _{n \rightarrow \infty} \mu_{n}\left(B_{j}\right)=\mu\left(B_{j}\right)$ for all $j$ with probability 1 . The almost sure convergence of $A_{n}(t)$ in countably many points of $\mathbb{R}_{+}$can be extended to all points in $\mathbb{R}_{+}$if the limit process $A(t)$ is almost surely continuous. In this case, $\lim _{n \rightarrow \infty} A_{n}(t)=A(t)$ with probability 1 for all $t \in \mathbb{R}_{+}$. As noted in Kahane (1987), there are two extreme cases: (i) $A_{n}(t) \rightarrow A(t)$ in $L_{1}$ for each given $t$, in which case $A(t)$ is not almost surely 0 and is said to be fully active (nondegenerate) on $\mathbb{R}_{+}$; (ii) $A_{n}$ (1) converges to 0 almost surely, in which case $A(t)$ is said to be degenerate on $\mathbb{R}_{+}$. Sufficient conditions for nondegeneracy and degeneracy in a general situation and relevant examples are provided in Kahane (1987, Equations (18) and (19), respectively). The condition for complete degeneracy is detailed in Theorem 3 of Kahane (1987).

The Rényi function, also known as the deterministic partition function, is defined for $t \in$ $[0,1]$ as

$$
\begin{aligned}
T(q) & =\liminf _{n \rightarrow \infty} \frac{\log \mathrm{E} \sum_{k=0}^{2^{n}-1} \mu^{q}\left(I_{k}^{(n)}\right)}{\log \left|I_{k}^{(n)}\right|} \\
& =\liminf _{n \rightarrow \infty}\left(-\frac{1}{n}\right) \log _{2} \mathrm{E} \sum_{k=0}^{2^{n}-1} \mu^{q}\left(I_{k}^{(n)}\right),
\end{aligned}
$$

where $I_{k}^{(n)}=\left[k 2^{-n},(k+1) 2^{-n}\right], k=0,1, \ldots, 2^{n}-1,\left|I_{k}^{(n)}\right|$ is its length, and $\log _{b}$ is the $\log$ to base $b$.

Remark 1. The multifractal formalism for random cascades can be stated in terms of the Legendre transform of the Rényi function:

$$
T^{*}(\alpha)=\min _{q \in \mathbb{R}}(q \alpha-T(q)) .
$$


In fact, let $f(\alpha)$ be the Hausdorff dimension of the set

$$
C_{\alpha}=\left\{t \in[0,1]: \lim _{n \rightarrow \infty} \frac{\log \mu\left(I_{k}^{(n)}(t)\right)}{\log \left|I_{k}^{(n)}\right|}=\alpha\right\},
$$

where $I_{k}^{(n)}(t)$ is a sequence of intervals $I_{k}^{(n)}$ that contain $t$. The function $f(\alpha)$ is known as the singularity spectrum of the measure $\mu$, and we refer to $\mu$ as a multifractal measure if $f(\alpha) \neq 0$ for a continuum of $\alpha$ (Lau (1999)). In order to determine the function $f(\alpha)$, Hentschel and Procaccia (1983), Frisch and Parisi (1985), and Halsey et al. (1986), for example, proposed to use the relationship

$$
f(\alpha)=T^{*}(\alpha)
$$

This relationship may not hold for a given measure (see, for example, Taylor (1995)). When equality (4) is established for a measure $\mu$, we say that the multifractal formalism holds for this measure.

Mannersalo et al. (2002) presented the conditions for $L_{2}$-convergence and scaling of moments.

Theorem 1. (Mannersalo et al. (2002).) Suppose that conditions (C1)-(C3) hold.

If, for some positive numbers $\delta$ and $\gamma$,

$$
\mathrm{e}^{-\delta|\tau|} \leq \rho(\tau)=\frac{M(1,1 ; \tau)-1}{M(2)-1} \leq|C \tau|^{-\gamma}
$$

then $A_{n}(t)$ converges in $L_{2}$ if and only if

$$
b>1+\sigma_{\Lambda}^{2}=M(2) .
$$

If $A_{n}(t)$ converges in $L_{1}$ then the limit process $A(t)$ satisfies the recursion

$$
A(t)=\frac{1}{b} \int_{0}^{t} \Lambda(s) \mathrm{d} \tilde{A}(b s),
$$

where the processes $\Lambda(t)$ and $\tilde{A}(t)$ are independent, and the processes $A(t)$ and $\tilde{A}(t)$ have identical finite-dimensional distributions.

If $A(t)$ is nondegenerate, recursion (6) holds, $A(1) \in L_{q}$ for some $q>0$, and

$$
\sum_{n=0}^{\infty} c\left(q, b^{-n}\right)<\infty
$$

where $c(q, t)=\operatorname{Esup}_{s \in[0, t]}\left|\Lambda^{q}(0)-\Lambda^{q}(s)\right|$, then there exist constants $\bar{C}$ and $\underline{C}$ such that

$$
\underline{C} t^{q-\log _{b} \mathrm{E} \Lambda^{q}(t)} \leq \mathrm{E} A^{q}(t) \leq \bar{C} t^{q-\log _{b} \mathrm{E} \Lambda^{q}(t)},
$$

which will be written as

$$
\mathrm{E} A^{q}(t) \sim t^{q-\log _{b} \mathrm{E} \Lambda^{q}(t)}, \quad t \in[0,1] .
$$

If, on the other hand, $A(1) \in L_{q}, q>1$, then the Rényi function is given by

$$
T(q)=q-1-\log _{b} \mathrm{E} \Lambda^{q}(t)=q-1-\log _{b} M(q) .
$$


If $A(t)$ is nondegenerate, $A(1) \in L_{2}$, and $\Lambda(t)$ is positively correlated, then

$$
\operatorname{var} A(t) \geq \operatorname{var} \int_{0}^{t} \Lambda(s) \mathrm{d} s .
$$

Hence, if $\int_{0}^{t} \Lambda(s) \mathrm{d} s$ is strongly dependent then $A(t)$ is also strongly dependent.

Remark 2. Result (7) means that the process $A(t), t \in[0,1]$, with stationary increments behaves as

$$
\log \mathrm{E}(A(t+\delta)-A(t))^{q} \sim K(q) \log \delta+C_{q}
$$

for a wide range of resolutions $\delta$ with a nonlinear function

$$
K(q)=q-\log _{b} \mathrm{E} \Lambda^{q}(t)=q-\log _{b} M(q),
$$

where $C_{q}$ is a constant. In this sense, the stochastic process $A(t)$ is said to be multifractal. The function $K(q)$, which contains the scaling parameter $b$ and all the parameters of the marginal distribution of the mother process $X(t)$, can be estimated by running regression (8) for a range of values of $q$. For the example in Section 4, the explicit form of $K(q)$ is obtained. Hence, these parameters can be estimated by minimising the mean square error between the $K(q)$ curve estimated from data and its analytical form for a range of values of $q$. This method has been used for multifractal characterisation of complete genomes in Anh et al. (2001).

\section{Infinitely divisible distributions and geometric Ornstein-Uhlenbeck processes}

In this section we review a number of known results on Lévy processes (see Skorokhod (1991), Bertoin (1996), Sato (1999), and Kyprianou (2006)) and Ornstein-Uhlenbeck-type processes (see Barndorff-Nielsen (2001), and Barndorff-Nielsen and Shephard (2001)). As standard notation, we will write

$$
\kappa(z)=C\{z ; X\}=\log \mathrm{Ee}^{\mathrm{i} z X}, \quad z \in \mathbb{R},
$$

for the cumulant function of a random variable $X$ and

$$
K\{\zeta ; X\}=\log \mathrm{Ee}^{\zeta X}, \quad \zeta \in \mathbb{R}
$$

for the Lévy exponent or Laplace transform or cumulant generating function of the random variable $X$. Its domain includes the imaginary axis and frequently larger areas.

A random variable $X$ is infinitely divisible if its cumulant function has the Lévy-Khintchine form

$$
C\{z ; X\}=\mathrm{i} a z-\frac{d}{2} z^{2}+\int_{\mathbb{R}}\left(\mathrm{e}^{\mathrm{i} z u}-1-\mathrm{i} z u \mathbf{1}_{[-1,1]}(u)\right) v(\mathrm{~d} u),
$$

where $a \in \mathbb{R}, d \geq 0$, and $v$ is the Lévy measure, that is, a nonnegative measure on $\mathbb{R}$ such that

$$
v(\{0\})=0, \quad \int_{\mathbb{R}} \min \left(1, u^{2}\right) v(\mathrm{~d} u)<\infty .
$$

The triplet $(a, d, v)$ uniquely determines the random variable $X$. For a Gaussian random variable, $X \sim N(a, d)$, the Lévy triplet takes the form $(a, d, 0)$.

A random variable $X$ is self-decomposable if, for all $c \in(0,1)$, the characteristic function $f(z)$ of $X$ can be factorised as $f(z)=f(c z) f_{c}(z)$ for some characteristic function $f_{c}(z), z \in \mathbb{R}$. 
Recall that a càdlàg stochastic process has right-continuous sample paths with existing left limits. A homogeneous Lévy process $Z=\{Z(t), t \geq 0\}$ is a continuous (in probability), càdlàg process with independent and stationary increments and $Z(0)=0$. For such processes, we have $C\{z ; Z(t)\}=t C\{z ; Z(1)\}$ and $Z(1)$ has the Lévy-Khintchine representation (9).

Let $f(z)$ be the characteristic function of a random variable $X$. If $X$ is self-decomposable then there exists a stationary stochastic process $\{X(t), t \geq 0\}$ such that $X(t) \stackrel{\mathrm{D}}{=} X$ and

$$
X(t)=\mathrm{e}^{-\lambda t} X(0)+\int_{(0, t]} \mathrm{e}^{-\lambda(t-s)} \mathrm{d} Z(\lambda s)
$$

for all $\lambda>0$ (see Barndorff-Nielsen (1998)). Conversely, if $\{X(t), t \geq 0\}$ is a stationary process and $\{Z(t), t \geq 0\}$ is a Lévy process, independent of $X(0)$, such that $X(t)$ and $Z(t)$ satisfy the Itô stochastic differential equation

$$
\mathrm{d} X(t)=-\lambda X(t) \mathrm{d} t+\mathrm{d} Z(\lambda t)
$$

for all $\lambda>0$, then $X(t)$ is self-decomposable. A stationary process $X(t)$ of this kind is said to be an Ornstein-Uhlenbeck-type process or an OU-type process for short. The process $Z(t)$ is termed the background driving Lévy process (BDLP) corresponding to the process $X(t)$. In fact, (10) is the unique (up to indistinguishability) strong solution to (11) (Sato (1999, Section 17)). The meaning of the stochastic integral in (10) was detailed in Applebaum (2004, p. 214).

A necessary and sufficient condition for (11) to have a stationary solution is that

$$
\mathrm{E} \log (1+|Z(1)|)<\infty .
$$

The stationary process $\{X(t), t \geq 0\}$ can be extended to a stationary process on the whole real line. To do this, we introduce an independent copy of the process $\{Z(t), t \geq 0\}$, but modify it to be càdlàg, thus obtaining a process $\{\bar{Z}(t), t \geq 0\}$, say. Now, for $t<0$, define $Z(t)=\bar{Z}(-t)$, and, for $t \in \mathbb{R}$, let

$$
X(t)=\mathrm{e}^{-\lambda t} \int_{-\infty}^{t} \mathrm{e}^{\lambda s} \mathrm{~d} Z(\lambda s) .
$$

Then, $\{Z(t), t \in \mathbb{R}\}$ is a homogeneous càdlàg Lévy process and $\{X(t), t \in \mathbb{R}\}$ is a strictly stationary process of OU-type.

Let $X(t)$ be a square-integrable OU process. Then $X(t)$ has the correlation function

$$
r_{X}(t)=\mathrm{e}^{-\lambda|t|}, \quad t \in \mathbb{R} .
$$

The cumulant transforms of $X=X(t)$ and $Z(1)$ are related by

$$
C\{z ; X\}=\int_{0}^{\infty} C\left\{\mathrm{e}^{-s} z ; Z(1)\right\} \mathrm{d} s=\int_{0}^{z} C\{\xi ; Z(1)\} \frac{\mathrm{d} \xi}{\xi}
$$

and

$$
C\{z ; Z(1)\}=z \frac{\partial C\{z ; X\}}{\partial z} .
$$

Suppose that the Lévy measure $v$ of $X$ has a density function $p(u), u \in \mathbb{R}$, which is differentiable. Then the Lévy measure $\tilde{v}$ of $Z(1)$ has a density function $q(u), u \in \mathbb{R}$, and $p$ and $q$ are related by

$$
q(u)=-p(u)-u p^{\prime}(u)
$$

(see Barndorff-Nielsen (1998)). 

form

The logarithm of the characteristic function of a random vector $\left(X\left(t_{1}\right), \ldots, X\left(t_{m}\right)\right)$ is of the

$$
\begin{aligned}
\log \mathrm{E} & \exp \left\{\mathrm{i}\left(z_{1} X\left(t_{1}\right)+\cdots+z_{m} X\left(t_{m}\right)\right)\right\} \\
= & \int_{\mathbb{R}} \kappa\left(\sum_{j=1}^{m} z_{j} \exp \left\{-\lambda\left(t_{j}-s\right)\right\} \mathbf{1}_{[0, \infty)}\left(t_{j}-s\right)\right) \mathrm{d} s,
\end{aligned}
$$

where

$$
\kappa(z)=\log \mathrm{E}^{\mathrm{i} z Z(1)}=C\{z ; Z(1)\},
$$

and function (15) has the form (9) with Lévy triplet $(\tilde{a}, \tilde{d}, \tilde{v})$ of $Z(1)$.

The logarithms of the moment generation functions (if they exist) take the forms

$$
\log M(\zeta)=\log \mathrm{E}^{\zeta X(t)}=\zeta a+\frac{d}{2} \zeta^{2}+\int_{\mathbb{R}}\left(\mathrm{e}^{\zeta u}-1-\zeta u \mathbf{1}_{[-1,1]}(u)\right) v(\mathrm{~d} u)
$$

where the triplet $(a, d, v)$ is the Lévy triplet of $X(0)$, or in terms of the Lévy triplet $(\tilde{a}, \tilde{d}, \tilde{v})$ of $Z$ (1),

$$
\begin{aligned}
\log M(\zeta)= & \tilde{a} \int_{\mathbb{R}}\left(\zeta \mathrm{e}^{-\lambda(t-s)} \mathbf{1}_{[0, \infty)}(t-s)\right) \mathrm{d} s+\frac{\tilde{d}}{2} \zeta^{2} \int_{\mathbb{R}}\left(\zeta \mathrm{e}^{-\lambda(t-s)} \mathbf{1}_{[0, \infty)}(t-s)\right)^{2} \mathrm{~d} s \\
+ & \int_{\mathbb{R}} \int_{\mathbb{R}}\left[\exp \left\{u \zeta \mathrm{e}^{-\lambda(t-s)} \mathbf{1}_{[0, \infty)}(t-s)\right\}-1\right. \\
& \left.-u\left(\zeta \mathrm{e}^{-\lambda(t-s)} \mathbf{1}_{[0, \infty)}(t-s)\right) \mathbf{1}_{[-1,1]}(u)\right] \tilde{v}(\mathrm{~d} u) \mathrm{d} s
\end{aligned}
$$

and

$$
\begin{aligned}
& \log M\left(\zeta_{1}, \zeta_{2} ; t_{1}-t_{2}\right) \\
& =\log \operatorname{Exp}\left\{\zeta_{1} X\left(t_{1}\right)+\zeta_{2} X\left(t_{2}\right)\right\} \\
& =\tilde{a} \int_{\mathbb{R}}\left(\sum_{j=1}^{2} \zeta_{j} \exp \left\{-\lambda\left(t_{j}-s\right)\right\} \mathbf{1}_{[0, \infty)}\left(t_{j}-s\right)\right) \mathrm{d} s \\
& \quad+\frac{\tilde{d}}{2} \zeta^{2} \int_{\mathbb{R}}\left(\sum_{j=1}^{2} \zeta_{j} \exp \left\{-\lambda\left(t_{j}-s\right)\right\} \mathbf{1}_{[0, \infty)}\left(t_{j}-s\right)\right)^{2} \mathrm{~d} s \\
& \quad+\int_{\mathbb{R}} \int_{\mathbb{R}}\left[\exp \left\{u \sum_{j=1}^{2} \zeta_{j} \exp \left\{-\lambda\left(t_{j}-s\right)\right\} \mathbf{1}_{[0, \infty)}\left(t_{j}-s\right)\right\}-1\right. \\
& \left.\quad-u\left(\sum_{j=1}^{2} \zeta_{j} \exp \left\{-\lambda\left(t_{j}-s\right)\right\} \mathbf{1}_{[0, \infty)}\left(t_{j}-s\right)\right) \mathbf{1}_{[-1,1]}(u)\right] \tilde{v}(\mathrm{~d} u) \mathrm{d} s
\end{aligned}
$$

The following result plays a key role in multifractal analysis of geometric OU processes.

Theorem 2. Let $X(t), t \in \mathbb{R}_{+}$, be an OU-type stationary process (10) such that the Lévy measure $v$ in (9) of the random variable $X(t)$ satisfies the condition that, for some $q \in Q \subseteq \mathbb{R}$,

$$
\int_{|x| \geq 1} g(x) v(\mathrm{~d} x)<\infty
$$


where $g(x)$ denotes any of the functions $\mathrm{e}^{2 q x}, \mathrm{e}^{q x}$, or $\mathrm{e}^{q x}|x|$. Then, for the geometric OU-type process, $\Lambda_{q}(t):=\mathrm{e}^{q X(t)}$,

$$
\sum_{n=0}^{\infty} c\left(q, b^{-n}\right)<\infty,
$$

where $c(q, t)=\operatorname{Esup}_{s \in[0, t]}\left|\Lambda_{q}(0)^{q}-\Lambda_{q}(s)^{q}\right|$.

To prove that our geometric OU-type process satisfies the covariance decay condition (5), the expression given by (18) is not ready to yield the decay as $t_{2}-t_{1} \rightarrow \infty$. The following proposition gives a general decay estimate which the driving Lévy processes $Z$ in Section 4 indeed satisfy.

Proposition 1. Consider an OU-type process $X$ given by

$$
\mathrm{d} X(t)=-\lambda X(t) \mathrm{d} t+\mathrm{d} Z(\lambda t)
$$

where the BDLP Z is without Gaussian part (that is, $\tilde{d}=0$ in (16)), and the Lévy measure $\tilde{v}(\mathrm{~d} x)$ of $Z$ has the density $g(x)$ for which there exists some $\beta>0$ such that $g(x) \leq($ constant $) \mathrm{e}^{-\beta|x|}$ for all $|x|>1$. Then there exist positive constants $c$ and $C$ such that

$$
\mathrm{E}\left(\mathrm{e}^{X(t)} \mathrm{e}^{X(0)}\right) \leq C \mathrm{e}^{-c t} \text { for all } t>0 .
$$

Remark 3. The constant $c$ is given by $\tilde{v}(|x|>1)$. If the region for the boundedness assumption on $g(x)$ is $|x|>a, a>1$, then $c$ is determined by $\tilde{v}(|x|>a)$.

The proofs of Theorem 2 and Proposition 1 will be given in Section 5.

Very often the correlation structure found in applications is more complex than the exponential decreasing autocorrelation of the form (12). Barndorff-Nielsen (1998), Barndorff-Nielsen and Sheppard (2001), and Barndorff-Nielsen and Leonenko (2005) proposed to consider the following class of autocovariance functions:

$$
R_{\text {sup }}(t)=\sum_{j=1}^{m} \sigma_{j}^{2} \exp \left\{-\lambda_{j}|t|\right\},
$$

which is flexible and can be fitted to many autocovariance functions arising in applications.

In order to obtain models with dependence structure (20) and given marginal density with finite variance, we consider stochastic processes defined by

$$
\mathrm{d} X_{j}(t)=-\lambda_{j} X_{j}(t) \mathrm{d} t+\mathrm{d} Z_{j}\left(\lambda_{j} t\right), \quad j=1,2, \ldots, m,
$$

and their superposition

$$
X_{\text {sup }}(t)=X_{1}(t)+\cdots+X_{m}(t), \quad t \geq 0,
$$

where the $Z_{j}, j=1,2, \ldots, m$, are mutually independent Lévy processes. Then the solution $X_{j}=\left\{X_{j}(t), t \geq 0\right\}, j=1,2, \ldots, m$, is a stationary process. Its correlation function is of the exponential form (assuming finite variance).

Superposition (21) has its marginal density given by that of the random variable

$$
X_{\text {sup }}(0)=X_{1}(0)+\cdots+X_{m}(0),
$$


autocovariance function (20) (where the $\sigma_{j}^{2}$ are now variances of $X_{j}$ ), and spectral density

$$
f_{\text {sup }}(\lambda)=\frac{2}{\pi} \sum_{j=1}^{m} \sigma_{j}^{2} \frac{\theta_{j}}{\theta_{j}+\lambda^{2}}, \quad \lambda \in \mathbb{R} .
$$

We are interested in the case when the distribution of (22) is tractable, for instance, when $X_{\text {sup }}(0)$ belongs to the same class as $X_{j}(0), j=1, \ldots, m$ (see the example in Section 4 ).

Note that an infinite superposition $(m \rightarrow \infty)$ gives a complete monotone class of covariance functions

$$
R_{\text {sup }}(t)=\int_{0}^{\infty} \mathrm{e}^{-t u} \mathrm{~d} U(u), \quad t \geq 0,
$$

for some finite measure $U$, which display long-range dependence (see Barndorff-Nielsen (1998), (2001) and Barndorff-Nielsen and Leonenko (2005) for possible covariance structures and spectral densities).

\section{Multifractal analysis of geometric OU-type processes}

In this section we introduce five illustrative multifractal scenarios. The mother process in this section will take the form

$$
\Lambda(t)=\exp \left\{X(t)-c_{X}\right\}
$$

where $X(t)$ is a stationary OU-type process (11) and $c_{X}$ is a constant depending on the parameters of its marginal distribution. This form is needed for the condition $\operatorname{E} \Lambda(t)=1$ to hold. Accordingly, the Rényi function is defined in this section as

$$
\begin{aligned}
T(q) & =q-1-\log _{b} \mathrm{E} \exp \left\{q\left(X(t)-c_{X}\right)\right\} \\
& =q\left(1+\frac{c_{X}}{\log b}\right)-\frac{1}{\log b} \log \mathrm{Ee}^{q X(t)}-1 .
\end{aligned}
$$

All the definitions given in (1)-(3) and, correspondingly, all the statements of Theorem 1 are now understood to be in terms of the mother process (23).

\subsection{Log-gamma scenario}

The log-gamma distribution is well known in the theory of turbulence and multiplicative cascades (Saito (1992)). In this section we propose a stationary version of the log-gamma scenario. We will use a stationary OU-type process (11) with marginal gamma distribution $\Gamma(\beta, \alpha)$, which is self-decomposable, and, hence, infinitely divisible. The probability density function (PDF) of $X(t), t \in \mathbb{R}_{+}$, is given by

$$
\pi(x)=\frac{\alpha^{\beta}}{\Gamma(\beta)} x^{\beta-1} \mathrm{e}^{-\alpha x} \mathbf{1}_{[0, \infty)}(x), \quad \alpha>0, \beta>0,
$$

with the Lévy triplet of the form $(0,0, v)$, where

$$
v(\mathrm{~d} u)=\frac{\beta \mathrm{e}^{-\alpha u}}{u} \mathbf{1}_{[0, \infty)}(u) \mathrm{d} u,
$$

while the BDLP $Z(t)$ in (11) is a compound Poisson subordinator

$$
Z(t)=\sum_{n=1}^{P(t)} Z_{n}
$$


with the $Z_{n}, n=1,2, \ldots$, being independent copies of the random variable $\Gamma(1, \alpha)$ and $P(t), t \geq 0$, being a homogeneous Poisson process with intensity $\beta$. The logarithm of the characteristic function of $Z(1)$ is

$$
\kappa(z)=\log \mathrm{E} \mathrm{e}^{\mathrm{i} z Z(1)}=\frac{\mathrm{i} \beta z}{\alpha-\mathrm{i} z}, \quad z \in \mathbb{R},
$$

and the (finite) Lévy measure $\tilde{v}$ of $Z(1)$ is

$$
\tilde{v}(\mathrm{~d} u)=\alpha \beta \mathrm{e}^{-\alpha u} \mathbf{1}_{(0, \infty)}(u) \mathrm{d} u .
$$

The correlation function is then

$$
r_{X}(t)=\mathrm{e}^{-\lambda|t|}, \quad t \in \mathbb{R} .
$$

(C4) Consider a mother process of the form

$$
\Lambda(t)=\exp \left\{X(t)-c_{X}\right\} \quad \text { with } \quad c_{X}=\log \frac{1}{(1-1 / \alpha)^{\beta}} \text { and } \alpha>1,
$$

where $X(t), t \in \mathbb{R}_{+}$, is a stationary gamma OU-type stochastic process with marginal density (24) and covariance function

$$
R_{X}(t)=\frac{\beta}{\alpha^{2}} \mathrm{e}^{-\lambda|t|}, \quad t \in \mathbb{R}
$$

Under condition (C4), we obtain the following moment generating function:

$$
M(\zeta)=\operatorname{E} \exp \left\{\zeta\left(X(t)-c_{X}\right)\right\}=\frac{\exp \left\{-c_{X} \zeta\right\}}{(1-\zeta / \alpha)^{\beta}}, \quad \zeta<\alpha, \alpha>1,
$$

and the bivariate moment generating function is given by (18), in which the measure $\tilde{v}$ is given by $(25)$, since

$$
\begin{aligned}
& M\left(\zeta_{1}, \zeta_{2} ;\left(t_{1}-t_{2}\right)\right) \\
& \quad=\operatorname{Exp}\left\{\zeta_{1}\left(X\left(t_{1}\right)-c_{X}\right)+\zeta_{2}\left(X\left(t_{2}\right)-c_{X}\right)\right\} \\
& \quad=\exp \left\{-c_{X}\left(\zeta_{1}+\zeta_{2}\right)\right\} \operatorname{E} \exp \left\{\zeta_{1} X\left(t_{1}\right)+\zeta_{2} X\left(t_{2}\right)\right\} \\
& \quad=\exp \left\{-c_{X}\left(\zeta_{1}+\zeta_{2}\right)\right\} \exp \left\{\int_{\mathbb{R}} \frac{\beta \sum_{j=1}^{2} \zeta_{j} \exp \left\{-\lambda\left(t_{j}-s\right)\right\} \mathbf{1}_{[0, \infty)}\left(t_{j}-s\right)}{\alpha-\sum_{j=1}^{2} \zeta_{j} \exp \left\{-\lambda\left(t_{j}-s\right)\right\} \mathbf{1}_{[0, \infty)}\left(t_{j}-s\right)} \mathrm{d} s\right\},
\end{aligned}
$$

or

$$
\begin{aligned}
& M\left(\zeta_{1}, \zeta_{2} ;\left(t_{1}-t_{2}\right)\right) \\
& =\exp \left\{-c_{X}\left(\zeta_{1}+\zeta_{2}\right)\right\} \\
& \times \exp \left\{\int _ { \mathbb { R } } \int _ { \mathbb { R } } \left(\exp \left\{u \sum_{j=1}^{2} \zeta_{j} \exp \left\{-\lambda\left(t_{j}-s\right)\right\} \mathbf{1}_{[0, \infty)}\left(t_{j}-s\right)\right\}-1\right.\right. \\
& \left.-u\left(\sum_{j=1}^{2} \zeta_{j} \exp \left\{-\lambda\left(t_{j}-s\right)\right\} \mathbf{1}_{[0, \infty)}\left(t_{j}-s\right)\right) \mathbf{1}_{[-1,1]}(u)\right) \\
& \left.\times \alpha \beta \mathrm{e}^{-\alpha u} \mathbf{1}_{(0, \infty)}(u) \mathrm{d} u \mathrm{~d} s\right\} .
\end{aligned}
$$


Thus, the correlation function of the mother process takes the form

$$
\rho(\tau)=\frac{M(1,1 ; \tau)-1}{M(2)-1},
$$

where $M(2)$ is given by $(26)$ and $M(1,1 ; \tau)$ is given by (28). It turns out that, in this case,

$$
\log _{b} \mathrm{E} \Lambda(t)^{q}=\frac{1}{\log b}\left(-q \log \frac{1}{(1-1 / \alpha)^{\beta}}-\beta \log \left(1-\frac{q}{\alpha}\right)\right),
$$

and condition (19) holds for $g(x)=\mathrm{e}^{q x}, q<\alpha$ :

$$
\int_{|u| \geq 1} \mathrm{e}^{q u} v(\mathrm{~d} u)=\int_{1}^{\infty} \mathrm{e}^{q u} \frac{\beta \mathrm{e}^{-\alpha u}}{u} \mathrm{~d} u<\infty .
$$

We formulate the following theorem.

Theorem 3. Suppose that condition (C4) holds, and let $Q=\{q: 0<q<\alpha, \alpha>2\}$. Then, for any $b>\exp \left\{-2 c_{X}\right\}(1-2 \alpha)^{-\beta}, \beta>0$, the stochastic processes $A_{n}(t)$ defined by (2) for the mother process (23) converge in $L_{2}$ to the stochastic process $A(t)$ as $n \rightarrow \infty$ such that, if $A(1) \in L_{q}$ for $q \in Q$,

$$
\mathrm{E} A(t)^{q} \sim t^{T(q)+1}
$$

where the Rényi function $T(q)$ is given by

$$
T(q)=q\left(1+\frac{1}{\log b} \log \frac{1}{(1-1 / \alpha)^{\beta}}\right)+\frac{\beta}{\log b} \log \left(1-\frac{q}{\alpha}\right)-1, \quad q \in Q .
$$

Moreover,

$$
\operatorname{var} A(t) \geq \int_{0}^{t} \int_{0}^{t}(M(1,1 ; u-w)-1) \mathrm{d} u \mathrm{~d} w
$$

where $M$ is given by (27) or (28).

Proof. Theorem 3 follows from Theorems 1 and 2 and Proposition 1.

Remark 4. For $q \in Q \cap[1,2]$, the condition $A(1) \in L_{q}, q>1$, is not needed; thus, the above results hold at least for this range. However, for $q$ outside this range, the condition is partly required for the validity of multifractal moment scaling. This remark also applies to all other scenarios in this section.

We can construct log-gamma scenarios for a more general class of finite superpositions of stationary gamma OU-type processes defined in (21), where the $X_{j}(t), j=1, \ldots, m$, are independent OU-type gamma stationary processes with marginals $\Gamma\left(\beta_{j}, \alpha\right), j=1, \ldots, m$, and parameters $\lambda_{j}, j=1, \ldots, m$. Then $X_{\text {sup }}(t), t \in \mathbb{R}_{+}$, has the marginal distribution $\Gamma\left(\sum_{j=1}^{m} \beta_{j}, \alpha\right)$ and covariance function

$$
R_{\text {sup }}(t)=\frac{1}{\alpha^{2}} \sum_{j=1}^{m} \beta_{j} \exp \left\{-\lambda_{j}|t|\right\}, \quad t \in \mathbb{R} .
$$

The generalisation of Theorem 2 and Proposition 1 to this situation is straightforward and the statement of Theorem 3 can be reformulated for the process of superposition $X_{\text {sup }}$ with $\beta=\sum_{j=1}^{m} \beta_{j}$ and $M_{\boldsymbol{\theta}}\left(\zeta_{1}, \zeta_{2} ;\left(t_{1}-t_{2}\right)\right)=\prod_{j=1}^{m} M_{\boldsymbol{\theta}_{j}}\left(\zeta_{1}, \zeta_{2} ;\left(t_{1}-t_{2}\right)\right)$, where $\boldsymbol{\theta}=(\alpha, \beta)$ and $\boldsymbol{\theta}_{j}=\left(\alpha, \beta_{j}\right)$, and $\lambda$ must be replaced by $\lambda_{j}$ in the expressions for $M_{\boldsymbol{\theta}}\left(\zeta_{1}, \zeta_{2} ;\left(t_{1}-t_{2}\right)\right),(27)$ or (28). Here, we insert the subscripts $\boldsymbol{\theta}$ and $\boldsymbol{\theta}_{j}$ in $\boldsymbol{M}_{\boldsymbol{\theta}}$ and $\boldsymbol{M}_{\boldsymbol{\theta}_{j}}$ to distinguish the parameter vectors. 


\subsection{Log-inverse Gaussian scenario}

In this section we propose a stationary version of the log-inverse Gaussian scenario. We will use a stationary OU-type process (11) with marginal inverse Gaussian distribution $\operatorname{IG}(\delta, \gamma)$ (see, for example, Barndorff-Nielsen and Sheppard (2001)), which is self-decomposable and, hence, infinitely divisible. The PDF of $X(t), t \in \mathbb{R}_{+}$, is given by

$$
\pi(x)=\frac{1}{\sqrt{2 \pi}} \frac{\delta \mathrm{e}^{\delta \gamma}}{x^{3 / 2}} \exp \left\{-\left(\frac{\delta^{2}}{x}+\gamma^{2} x\right) \frac{1}{2}\right\} \mathbf{1}_{[0, \infty)}(x), \quad \delta>0, \gamma \geq 0,
$$

with the Lévy triplet of the form $(0,0, v)$, where

$$
v(\mathrm{~d} u)=\frac{1}{\sqrt{2 \pi}} \frac{\delta}{u^{3 / 2}} \exp \left\{-\frac{\gamma^{2} u}{2}\right\} \mathbf{1}_{[0, \infty)}(u) \mathrm{d} u,
$$

while the BDLP $Z(t)$ in (11) has the cumulant transform

$$
\kappa(z)=\log \mathrm{Ee}^{\mathrm{i} z Z(1)}=\frac{\mathrm{i} z \delta}{\gamma \sqrt{1-2 \mathrm{i} z / \gamma^{2}}}, \quad z \in \mathbb{R},
$$

that is, the Lévy triplet of $Z(1)$ is of the form $(0,0, \tilde{v})$, and $Z(t)$ is the sum of two independent Lévy processes: $Z(t)=Z_{1}(t)+Z_{2}(t)$. Here $Z_{1}(t), t \in \mathbb{R}_{+}$, is an $\operatorname{IG}(\delta / 2, \gamma)$ subordinator with Lévy density

$$
\tilde{v}_{1}(\mathrm{~d} u)=\frac{1}{2 \sqrt{2 \pi}} \frac{\delta}{u \sqrt{u}} \exp \left\{-\frac{\gamma^{2} u}{2}\right\} \mathbf{1}_{[0, \infty)}(u) \mathrm{d} u,
$$

which has infinitely many jumps in bounded time intervals, and $Z_{2}(t), t \in \mathbb{R}_{+}$, is a compound Poisson subordinator:

$$
Z_{2}(t)=\frac{1}{\gamma^{2}} \sum_{n=1}^{P(t)} Z_{n}^{2}
$$

where the $Z_{n}, n=1,2, \ldots$, are independent copies of the standard normal variable and $P(t), t \in \mathbb{R}_{+}$, is a homogeneous Poisson process with intensity $\delta \gamma / 2$. The (finite) Lévy measure $\tilde{v}$ of $Z_{2}(1)$ can be computed as

$$
\tilde{v}_{2}(\mathrm{~d} u)=\frac{1}{2 \sqrt{2 \pi}} \frac{\delta \gamma^{2}}{\sqrt{u}} \exp \left\{-\frac{\gamma^{2} u}{2}\right\} \mathbf{1}_{[0, \infty)}(u) \mathrm{d} u .
$$

The correlation function is then

$$
r_{X}(t)=\mathrm{e}^{-\lambda|t|}, \quad t \in \mathbb{R} .
$$

(C5) Consider a mother process of the form

$$
\Lambda(t)=\exp \left\{X(t)-c_{X}\right\} \quad \text { with } \quad c_{X}=\delta\left(\gamma-\sqrt{\gamma^{2}-2}\right) \text { and } \gamma \geq \sqrt{2}
$$

where $X(t), t \in \mathbb{R}_{+}$, is a stationary inverse Gaussian OU-type process with marginal density (29) and covariance function

$$
R_{X}(t)=\frac{\delta}{\gamma^{3}} \mathrm{e}^{-\lambda|t|}, \quad t \in \mathbb{R} .
$$


Under condition (C5), we obtain the following moment generating function:

$$
M(\zeta)=\operatorname{Eexp}\left\{\zeta\left(X(t)-c_{X}\right)\right\}=\exp \left\{-c_{X} \zeta+\delta\left(\gamma-\sqrt{\gamma^{2}-2 \zeta}\right)\right\}, \quad \zeta<\frac{\gamma^{2}}{2}, \gamma \geq \sqrt{2},
$$

and the bivariate moment generating function is given by (18), in which the measure $\tilde{v}$ is given by (30) and (31) since

$$
\begin{aligned}
M\left(\zeta_{1}, \zeta_{2}\right. & \left.;\left(t_{1}-t_{2}\right)\right) \\
= & \exp \left\{\zeta_{1}\left(X\left(t_{1}\right)-c_{X}\right)+\zeta_{2}\left(X\left(t_{2}\right)-c_{X}\right)\right\} \\
= & \exp \left\{-c_{X}\left(\zeta_{1}+\zeta_{2}\right)\right\} \operatorname{E} \exp \left\{\zeta_{1} X\left(t_{1}\right)+\zeta_{2} X\left(t_{2}\right)\right\} \\
= & \exp \left\{-c_{X}\left(\zeta_{1}+\zeta_{2}\right)\right\} \\
& \times \exp \left\{\int_{\mathbb{R}} \frac{\delta \sum_{j=1}^{2} \zeta_{j} \exp \left\{-\lambda\left(t_{j}-s\right)\right\} \mathbf{1}_{[0, \infty)}\left(t_{j}-s\right)}{\gamma \sqrt{1-2 \beta \sum_{j=1}^{2} \zeta_{j} \exp \left\{-\lambda\left(t_{j}-s\right)\right\} \mathbf{1}_{[0, \infty)}\left(t_{j}-s\right) / \gamma^{2}}} \mathrm{~d} s\right\},
\end{aligned}
$$

or

$$
\begin{aligned}
& M\left(\zeta_{1}, \zeta_{2} ;\left(t_{1}-t_{2}\right)\right) \\
& =\exp \left\{-c_{X}\left(\zeta_{1}+\zeta_{2}\right)\right\} \\
& \quad \times \exp \left\{\int _ { \mathbb { R } } \int _ { \mathbb { R } } \left(\exp \left\{u \sum_{j=1}^{2} \zeta_{j} \exp \left\{-\lambda\left(t_{j}-s\right)\right\} \mathbf{1}_{[0, \infty)}\left(t_{j}-s\right)\right\}-1\right.\right. \\
& \left.\quad-u\left(\sum_{j=1}^{2} \zeta_{j} \exp \left\{-\lambda\left(t_{j}-s\right)\right\} \mathbf{1}_{[0, \infty)}\left(t_{j}-s\right)\right) \mathbf{1}_{[-1,1]}(u)\right) \\
& \left.\quad \times\left(\frac{1}{2 \sqrt{2 \pi}} \frac{\delta}{u \sqrt{u}} \exp \left\{-\frac{\gamma^{2} u}{2}\right\}+\frac{1}{2 \sqrt{2 \pi}} \frac{\delta \gamma^{2}}{\sqrt{u}} \exp \left\{-\frac{\gamma^{2} u}{2}\right\}\right) \mathbf{1}_{(0, \infty)}(u) \mathrm{d} u \mathrm{~d} s\right\}
\end{aligned}
$$

Thus, the correlation function of the mother process takes the form

$$
\rho(\tau)=\frac{M(1,1 ; \tau)-1}{M(2)-1}
$$

where $M(2)$ is given by $(32)$ and $M(1,1 ; \tau)$ is given by (34). In this case,

$$
\log _{b} \mathrm{E} \Lambda(t)^{q}=\frac{1}{\log b}\left(-q\left(\delta\left(\gamma-\sqrt{\gamma^{2}-2}\right)\right)+\delta\left(\left(\gamma-\sqrt{\gamma^{2}-2 q}\right)\right)\right),
$$

and condition (19) holds for $q<\gamma^{2} / 2$ with

$$
\int_{|u| \geq 1} \mathrm{e}^{q u} v(\mathrm{~d} u)=\int_{1}^{\infty} \mathrm{e}^{q u} \frac{1}{\sqrt{2 \pi}} \frac{\delta}{u^{3 / 2}} \exp \left\{-\frac{\gamma^{2} u}{2}\right\} \mathrm{d} u<\infty .
$$

We formulate the following theorem.

Theorem 4. Suppose that condition (C5) holds, and let $Q=\left\{q: 0<q<\gamma^{2} / 2, \gamma \geq 2\right\}$. Then, for any $b>\exp \left\{-2 c_{X}+\delta\left(\gamma-\sqrt{\gamma^{2}-4}\right)\right\}$, the stochastic processes $A_{n}(t)$ defined by $(2)$ 
for the mother process (23) converge in $L_{2}$ to the stochastic process $A(t)$ as $n \rightarrow \infty$ such that, if $A(1) \in L_{q}$ for $q \in Q$,

$$
\mathrm{E} A(t)^{q} \sim t^{T(q)+1}
$$

where the Rényi function is given by

$$
T(q)=q\left(1+\frac{\delta\left(\gamma-\sqrt{\gamma^{2}-2}\right)}{\log b}\right)+\frac{\delta}{\log b} \sqrt{\gamma^{2}-2 q}-\frac{\gamma \delta}{\log b}-1 .
$$

Moreover,

$$
\operatorname{var} A(t) \geq \int_{0}^{t} \int_{0}^{t}(M(1,1 ; u-w)-1) \mathrm{d} u \mathrm{~d} w
$$

where $M$ is given by (33) or (34).

Proof. Theorem 4 follows from Theorems 1 and 2 and Proposition 1.

We can construct log-inverse Gaussian scenarios for a more general class of finite superpositions of stationary inverse Gaussian OU-type processes defined by (21), where the $X_{j}(t), j=1, \ldots, m$, are independent inverse Gaussian stationary processes with marginals $\mathrm{IG}\left(\delta_{j}, \gamma\right), j=1, \ldots, m$, and parameters $\lambda_{j}, j=1, \ldots, m$. Then $X_{\text {sup }}(t), t \in \mathbb{R}_{+}$, has the marginal distribution $\operatorname{IG}\left(\sum_{j=1}^{m} \delta_{j}, \gamma\right)$ and covariance function

$$
R_{\text {sup }}(t)=\frac{1}{\gamma^{3}} \sum_{j=1}^{m} \delta_{j} \exp \left\{-\lambda_{j}|t|\right\}, \quad t \in \mathbb{R} .
$$

The generalisation of Theorem 2 and Proposition 1 to this situation is straightforward and the statement of Theorem 4 can be reformulated for the process of superposition $X_{\text {sup }}$ with $\delta=\sum_{j=1}^{m} \delta_{j}$ and $M_{\boldsymbol{\theta}}\left(\zeta_{1}, \zeta_{2} ;\left(t_{1}-t_{2}\right)\right)=\prod_{j=1}^{m} M_{\boldsymbol{\theta}_{j}}\left(\zeta_{1}, \zeta_{2} ;\left(t_{1}-t_{2}\right)\right)$, where $\boldsymbol{\theta}=(\delta, \gamma)$ and $\boldsymbol{\theta}_{j}=\left(\delta_{j}, \gamma\right)$, and $\lambda$ must be replaced by $\lambda_{j}$ in the expressions for $M_{\boldsymbol{\theta}_{j}}\left(\zeta_{1}, \zeta_{2} ;\left(t_{1}-t_{2}\right)\right)$, (33) or (34).

\subsection{Log-normal inverse Gaussian scenario}

We need the modified Bessel function of the third kind of index $\lambda$ :

$$
K_{\lambda}(z)=\int_{0}^{\infty} \mathrm{e}^{-z \cosh (x)} \cosh (\lambda x) \mathrm{d} x, \quad \operatorname{Re} \lambda>0 .
$$

Note that, as $z \rightarrow \infty$

$$
K_{\lambda}(z)=\sqrt{\frac{\pi}{2}} z^{-1 / 2} \mathrm{e}^{-z}\left(1+\frac{4 \lambda^{2}-1}{8 z}+\cdots\right), \quad z>0 .
$$

We will use a stationary OU-type process (11) with marginal NIG distribution $\mathrm{NIG}(\alpha, \beta, \delta, \mu)$, which is self-decomposable and, hence, infinitely divisible. This process was first introduced and studied in Barndorff-Nielsen (1998). The PDF of $X(t), t \in \mathbb{R}_{+}$, is of the form

$$
\pi(x)=\frac{\alpha \delta}{\pi} \mathrm{e}^{\delta \gamma} \frac{K_{1}\left(\alpha \sqrt{\delta^{2}+(x-\mu)^{2}}\right)}{\sqrt{\delta^{2}+(x-\mu)^{2}}} \mathrm{e}^{\beta(x-\mu)}, \quad x \in \mathbb{R},
$$

where $K_{\lambda}(z)$ is defined by (35) and the set of parameters is

$$
\delta>0, \quad 0 \leq|\beta| \leq \alpha, \quad \mu \in \mathbb{R}, \quad \text { and } \quad \gamma^{2}=\alpha^{2}-\beta^{2} .
$$


This distribution is symmetric around $\mu$ provided that $\beta=0$. Note that

$$
\mathrm{E} X(t)=\mu+\frac{\delta \beta}{\gamma}, \quad \operatorname{var} X(t)=\frac{\delta \alpha^{2}}{\gamma^{3}},
$$

and $\operatorname{NIG}(\alpha, \beta, \delta, \mu)$ has semiheavy tails, specifically,

$$
\operatorname{NIG}(u) \sim(\text { constant })|u|^{-3 / 2} \exp \{-\alpha|u|+\beta u\} \quad \text { as } u \rightarrow \pm \infty .
$$

The moment generating function of $\operatorname{NIG}(\alpha, \beta, \delta, \mu)$ is

$$
K\{\zeta ; X(t)\}=\mu \zeta+\delta\left(\sqrt{\alpha^{2}-\beta^{2}}-\sqrt{\alpha^{2}-(\beta+\zeta)^{2}}\right), \quad|\beta+\zeta|<\alpha .
$$

Thus, if the $X_{j}(t), j=1, \ldots, m$, are independent so that $X_{j} \sim \operatorname{NIG}\left(\alpha, \beta, \delta_{j}, \mu_{j}\right), j=$ $1, \ldots, m$, then we have

$$
X_{1}(t)+\cdots+X_{m}(t) \sim \operatorname{NIG}\left(\alpha, \beta, \delta_{1}+\cdots+\delta_{n}, \mu_{1}+\cdots+\mu_{n}\right) .
$$

The Lévy triplet $X(t)$ is of the form $(a, 0, v)$, where

$$
\begin{gathered}
a=\mu+2 \pi^{-1} \delta \alpha \int_{0}^{1} \sinh (\beta x) K_{1}(\alpha x) \mathrm{d} x, \\
v(\mathrm{~d} u)=p(u) \mathrm{d} u=\pi^{-1} \delta \alpha|u|^{-1} K_{1}(\alpha|u|) \mathrm{e}^{\beta u} \mathrm{~d} u .
\end{gathered}
$$

Note that, for $\operatorname{NIG}(\alpha, 0,1,0)$, we have

$$
p(u)=\pi^{-1} \delta \alpha|u|^{-1} K_{1}(\alpha|u|),
$$

while the $\operatorname{BDLP} Z(t)$ in (11) has a Lévy measure $\tilde{v}$ with density

$$
q(u)=(1-\beta u) \pi^{-1} \delta \alpha|u|^{-1} K_{1}(\alpha|u|) \mathrm{e}^{\beta u}+\pi^{-1} \alpha^{2} \delta K_{0}(\alpha|u|) \mathrm{e}^{\beta u} .
$$

The correlation function of the stationary process with marginal density (36) is then

$$
r_{X}(t)=\mathrm{e}^{-\lambda|t|}, \quad t \in \mathbb{R} .
$$

(C6) Consider a mother process of the form

$$
\begin{gathered}
\Lambda(t)=\exp \left\{X(t)-c_{X}\right\}, \\
\text { with } c_{X}=\mu+\delta \sqrt{\alpha^{2}-\beta^{2}}-\sqrt{\alpha^{2}-(\beta+1)^{2}} \text { and }|\beta+1|<\alpha,
\end{gathered}
$$

where $X(t), t \in \mathbb{R}_{+}$, is a stationary $\operatorname{NIG}(\alpha, \beta, \delta, \mu)$ OU-type process with marginal density (36) and covariance function

$$
R_{X}(t)=\frac{\delta \alpha^{2}}{\gamma^{3}} \mathrm{e}^{-\lambda|t|}, \quad t \in \mathbb{R} .
$$

Under condition (C6), we obtain the following moment generating function:

$$
\begin{aligned}
M(\zeta) & =\operatorname{E} \exp \left\{\zeta\left(X(t)-c_{X}\right)\right\} \\
& =\exp \left\{-c_{X} \zeta\right\} \exp \left\{\mu \zeta+\delta\left(\sqrt{\alpha^{2}-\beta^{2}}-\sqrt{\alpha^{2}-(\beta+\zeta)^{2}}\right)\right\}, \quad|\beta+\zeta|<\alpha
\end{aligned}
$$


and the bivariate moment generating function

$$
\begin{aligned}
M\left(\zeta_{1}, \zeta_{2} ;\left(t_{1}-t_{2}\right)\right) & =\operatorname{E} \exp \left\{\zeta_{1}\left(X\left(t_{1}\right)-c_{X}\right)+\zeta_{2}\left(X\left(t_{2}\right)-c_{X}\right)\right\} \\
& =\exp \left\{-c_{X}\left(\zeta_{1}+\zeta_{2}\right)\right\} \operatorname{E} \exp \left\{\zeta_{1} X\left(t_{1}\right)+\zeta_{2} X\left(t_{2}\right)\right\}
\end{aligned}
$$

where $\operatorname{E} \exp \left\{\zeta_{1} X\left(t_{1}\right)+\zeta_{2} X\left(t_{2}\right)\right\}$ is given by (16) with Lévy measure $\tilde{v}$ having density (37). Thus, the correlation function of the mother process takes the form

$$
\rho(\tau)=\frac{M(1,1 ; \tau)-1}{M(2)-1},
$$

where $M(2)$ is given by (38) and $M(1,1 ; \tau)$ is given by (39).

Condition (19) holds for $q<\alpha-|\beta|$ with

$$
\int_{|u| \geq 1} \mathrm{e}^{q u} v(\mathrm{~d} u)=\int_{|u| \geq 1} \mathrm{e}^{q u} \pi^{-1} \delta \alpha|u|^{-1} K_{1}(\alpha|u|) \mathrm{e}^{\beta u} \mathrm{~d} u<\infty .
$$

We formulate the following theorem.

Theorem 5. Suppose that condition (C6) holds, and let $Q=\{q$ : $0<q<\alpha-|\beta|,|\beta+1|<$ $\alpha,|\beta+2|<\alpha\}$. Then, for any

$$
b>\exp \left\{-2 c_{X}\right\} \exp \left\{2 \mu+\delta\left(\sqrt{\alpha^{2}-\beta^{2}}-\sqrt{\alpha^{2}-(\beta+2)^{2}}\right)\right\},
$$

the stochastic processes defined by (2) for the mother process (23) converge in $L_{2}$ to the stochastic process $A(t)$ as $n \rightarrow \infty$ such that, if $A(1) \in L_{q}$ for $q \in Q$,

$$
\mathrm{E} A(t)^{q} \sim t^{T(q)+1}
$$

where the Rényi function is given by

$$
\begin{aligned}
T(q)= & q\left(1+\frac{1}{\log b} \delta \sqrt{\alpha^{2}-\beta^{2}}-\frac{1}{\log b} \sqrt{\alpha^{2}-(1+\beta)^{2}}\right) \\
& +\frac{\delta}{\log b} \sqrt{\alpha^{2}-(q+\beta)^{2}}-\delta \frac{\sqrt{\alpha^{2}-\beta^{2}}}{\log b}-1 .
\end{aligned}
$$

Moreover,

$$
\operatorname{var} A(t) \geq \int_{0}^{t} \int_{0}^{t}(M(1,1 ; u-w)-1) \mathrm{d} u \mathrm{~d} w,
$$

where $M$ is given by (39).

Proof. Theorem 5 follows from Theorems 1 and 2 and Proposition 1.

We can construct log-normal inverse Gaussian scenarios for a more general class of finite superpositions of stationary normal inverse Gaussian OU-type processes defined by (21), where the $X_{j}(t), j=1, \ldots, m$, are independent normal inverse Gaussian stationary processes with marginals $\operatorname{NIG}\left(\alpha, \beta, \delta_{j}, \mu_{j}\right), j=1, \ldots, m$, and parameters $\lambda_{j}, j=1, \ldots, m$. Then $X_{\text {sup }}(t), t \in \mathbb{R}_{+}$, has the marginal distribution $\operatorname{NIG}\left(\alpha, \beta, \sum_{j=1}^{m} \delta_{j}, \sum_{j=1}^{m} \mu_{j}\right)$ and covariance function

$$
R_{\text {sup }}(t)=\frac{\alpha^{2}}{\gamma^{3}} \sum_{j=1}^{m} \delta_{j} \exp \left\{-\lambda_{j}|t|\right\}, \quad t \in \mathbb{R} .
$$


The generalisation of Theorem 2 and Proposition 1 to this situation is straightforward and the statement of Theorem 5 can be reformulated for the process of superposition $X_{\text {sup }}$ with $\delta=\sum_{j=1}^{m} \delta_{j}, \mu=\sum_{j=1}^{m} \mu_{j}$, and $M_{\boldsymbol{\theta}}\left(\zeta_{1}, \zeta_{2} ;\left(t_{1}-t_{2}\right)\right)=\prod_{j=1}^{m} M_{\boldsymbol{\theta}_{j}}\left(\zeta_{1}, \zeta_{2} ;\left(t_{1}-t_{2}\right)\right)$, where $\boldsymbol{\theta}=(\alpha, \beta, \delta, \mu)$ and $\boldsymbol{\theta}_{j}=\left(\alpha, \beta, \delta_{j}, \mu_{j}\right)$, and $\lambda$ must be replaced by $\lambda_{j}$ in the expression for $M_{\boldsymbol{\theta}_{j}}\left(\zeta_{1}, \zeta_{2} ;\left(t_{1}-t_{2}\right)\right),(39)$.

\subsection{Log-variance gamma scenario}

The next example of a hyperbolic OU process is based on the variance-gamma distribution (see, for example, Madan et al. (1998), Finlay and Seneta (2006), and Carr et al. (2007)). We will use a stationary OU-type process (11) with marginal variance gamma distribution $\operatorname{VG}(\lambda, \alpha, \beta, \mu)$, which is self-decomposable and, hence, infinitely divisible. The PDF of $X(t), t \in \mathbb{R}_{+}$, is

$$
\pi(x)=\frac{\gamma^{2 \kappa}}{\sqrt{\pi} \Gamma(\kappa)(2 \alpha)^{\kappa-1 / 2}}|x-\mu|^{\kappa-1 / 2} K_{\kappa-1 / 2}(\alpha|x-\mu|) \mathrm{e}^{\beta(x-\mu)}, \quad x \in \mathbb{R},
$$

where $K_{\lambda}(z)$ is defined by (35) and the set of parameters is

$$
\gamma^{2}=\alpha^{2}-\beta^{2}, \quad \kappa>0, \quad \alpha>|\beta|>0, \quad \text { and } \quad \mu \in \mathbb{R} .
$$

Note that

$$
\mathrm{E} X(t)=\mu+\frac{2 \beta \kappa}{\gamma^{2}}, \quad \operatorname{var} X(t)=\frac{2 \kappa}{\gamma^{2}}\left(1+2\left(\frac{\beta}{\gamma}\right)^{2}\right),
$$

and $\operatorname{VG}(\kappa, \alpha, \beta, \mu)$ has semiheavy tails. The moment generating function of $\operatorname{VG}(\kappa, \alpha, \beta, \mu)$ is

$$
K\{\zeta ; X(t)\}=\mu \zeta+2 \kappa \log \left(\frac{\gamma}{\sqrt{\alpha^{2}-(\beta+\zeta)^{2}}}\right), \quad|\beta+\zeta|<\alpha .
$$

Thus, if the $X_{j}(t), j=1, \ldots, m$, are independent so that $X_{j} \sim \operatorname{VG}\left(\kappa_{j}, \alpha, \beta, \mu_{j}\right), j=$ $1, \ldots, m$, then we have

$$
X_{1}(t)+\cdots+X_{m}(t) \sim \operatorname{VG}\left(\kappa_{1}+\cdots+\kappa_{n}, \alpha, \beta, \mu_{1}+\cdots+\mu_{n}\right) .
$$

The Lévy measure $v$ of $X(t)$ has density

$$
p(u)=\frac{\kappa}{|u|} \mathrm{e}^{\beta u-\alpha|u|}, \quad u \in \mathbb{R} .
$$

By (13), the Lévy measure $\tilde{v}$ of the BDLP $Z(t)$ in (11) has density

$$
\begin{gathered}
q(u)=-p(u)-u p^{\prime}(u), \\
\text { where } \quad p^{\prime}(u)= \begin{cases}-\frac{\kappa}{u} \mathrm{e}^{u(\beta+\alpha)}(\beta+\alpha)+\frac{\kappa}{u^{2}} \mathrm{e}^{u(\beta+\alpha)}, & u<0, \\
\frac{\kappa}{u} \mathrm{e}^{u(\beta-\alpha)}(\beta-\alpha)-\frac{\kappa}{u^{2}} \mathrm{e}^{u(\beta-\alpha)}, & u>0 .\end{cases}
\end{gathered}
$$

The correlation function of the stationary process with marginal density (40) is then

$$
r_{X}(t)=\mathrm{e}^{-\lambda|t|}, \quad t \in \mathbb{R} .
$$


(C7) Consider a mother process of the form

$$
\begin{gathered}
\Lambda(t)=\exp \left\{X(t)-c_{X}\right\}, \\
\text { with } \quad c_{X}=\mu+2 \kappa \log \left(\frac{\gamma}{\sqrt{\alpha^{2}-(\beta+1)^{2}}}\right) \text { and }|\beta+1|<\alpha,
\end{gathered}
$$

where $X(t), t \in \mathbb{R}_{+}$, is a stationary $\operatorname{VG}(\kappa, \alpha, \beta, \mu)$ OU-type process with marginal density (39) and covariance function

$$
R_{X}(t)=\frac{2 \kappa}{\gamma^{2}}\left(1+2\left(\frac{\beta}{\gamma}\right)^{2}\right) \mathrm{e}^{-\lambda|t|}, \quad t \in \mathbb{R} .
$$

Under condition (C7), we obtain the moment generating function

$$
\begin{aligned}
M(\zeta) & =\operatorname{E} \exp \left\{\zeta\left(X(t)-c_{X}\right)\right\} \\
& =\exp \left\{-c_{X} \zeta\right\} \exp \left\{\mu \zeta+2 \kappa \log \left(\frac{\gamma}{\sqrt{\alpha^{2}-(\beta+\zeta)^{2}}}\right)\right\}, \quad|\beta+\zeta|<\alpha,
\end{aligned}
$$

and the bivariate moment generating function

$$
\begin{aligned}
M\left(\zeta_{1}, \zeta_{2} ;\left(t_{1}-t_{2}\right)\right) & =\operatorname{Eexp}\left\{\zeta_{1}\left(X\left(t_{1}\right)-c_{X}\right)+\zeta_{2}\left(X\left(t_{2}\right)-c_{X}\right)\right\} \\
& =\exp \left\{-c_{X}\left(\zeta_{1}+\zeta_{2}\right)\right\} \operatorname{E} \exp \left\{\zeta_{1} X\left(t_{1}\right)+\zeta_{2} X\left(t_{2}\right)\right\},
\end{aligned}
$$

where $\operatorname{E} \exp \left\{\zeta_{1} X\left(t_{1}\right)+\zeta_{2} X\left(t_{2}\right)\right\}$ is given by (16) with Lévy measure $\tilde{v}$ having density (41). Thus, the correlation function of the mother process takes the form

$$
\rho(\tau)=\frac{M(1,1 ; \tau)-1}{M(2)-1},
$$

where $M(2)$ is given by $(41)$ and $M(1,1 ; \tau)$ is given by (42).

Condition (19) holds for $q<\alpha-|\beta|$. We formulate the following theorem.

Theorem 6. Suppose that condition (C7) holds, and let $Q=\{q: 0<q<\alpha-|\beta|,|\beta+1|<$ $\alpha,|\beta+2|<\alpha\}$. Then, for any

$$
b>\exp \left\{-2 c_{X}\right\} \exp \left\{\mu 2+2 \kappa \log \left(\frac{\gamma}{\sqrt{\alpha^{2}-(\beta+2)^{2}}}\right)\right\},
$$

the stochastic processes $A_{n}(t)$ defined by (2) for the mother process (23) converge in $L_{2}$ to the stochastic process $A(t)$ as $n \rightarrow \infty$ such that, if $A(1) \in L_{q}$ for $q \in Q$,

$$
\mathrm{E} A(t)^{q} \sim t^{T(q)+1}
$$

where the Rényi function is given by

$$
\begin{aligned}
T(q)= & q\left(1+\frac{2 \kappa}{\log b} \log \frac{\gamma}{\sqrt{\alpha^{2}-(\beta+1)^{2}}}\right) \\
& -\frac{2 \kappa}{\log b} \log \sqrt{\alpha^{2}-(\beta+q)^{2}}-\frac{2 \kappa}{\log b} \log \gamma-1 .
\end{aligned}
$$

Moreover,

$$
\operatorname{var} A(t) \geq \int_{0}^{t} \int_{0}^{t}(M(1,1 ; u-w)-1) \mathrm{d} u \mathrm{~d} w,
$$

where $M$ is given by (42). 
Proof. Theorem 6 follows from Theorems 1 and 2 and Proposition 1.

We can construct log-variance gamma scenarios for a more general class of finite superpositions of stationary variance gamma OU-type processes of the form (21), where the $X_{j}(t), j=1, \ldots, m$, are independent variance gamma stationary processes with marginals $X_{j} \sim \operatorname{VG}\left(\kappa_{j}, \alpha, \beta, \mu_{j}\right), j=1, \ldots, m$, and parameters $\lambda_{j}, j=1, \ldots, m$. Then $X_{\text {sup }}(t)$, $t \in \mathbb{R}_{+}$, has the marginal distribution $\operatorname{VG}\left(\kappa_{1}+\cdots+\kappa_{m}, \alpha, \beta, \delta, \mu_{1}+\cdots+\mu_{m}\right)$ and covariance function

$$
R_{\text {sup }}(t)=\frac{2}{\gamma^{2}}\left(1+2\left(\frac{\beta}{\gamma}\right)^{2}\right) \sum_{j=1}^{m} \kappa_{j} \exp \left\{-\lambda_{j}|t|\right\}, \quad t \in \mathbb{R} .
$$

The generalisation of Theorem 2 and Proposition 1 to this situation is straightforward and the statement of Theorem 6 can be reformulated for the process of superposition $X_{\text {sup }}$ with $\lambda=\sum_{j=1}^{m} \lambda_{j}, \mu=\sum_{j=1}^{m} \mu_{j}$, and $M_{\boldsymbol{\theta}}\left(\zeta_{1}, \zeta_{2} ;\left(t_{1}-t_{2}\right)\right)=\prod_{j=1}^{m} M_{\boldsymbol{\theta}_{j}}\left(\zeta_{1}, \zeta_{2} ;\left(t_{1}-t_{2}\right)\right)$, where $\boldsymbol{\theta}=(\kappa, \beta, \delta, \mu)$ and $\boldsymbol{\theta}_{j}=\left(\kappa_{j}, \alpha, \beta, \mu_{j}\right)$, and $\lambda$ must be replaced by $\lambda_{j}$ in the expression for $M_{\boldsymbol{\theta}_{j}}\left(\zeta_{1}, \zeta_{2} ;\left(t_{1}-t_{2}\right)\right),(39)$.

\subsection{Log-z scenario}

The next scenario is based on the $z$-distribution (see, for example, Grigelionis (2001)). We consider a PDF of the form

$$
\pi(x)=\frac{2 \pi \exp \left\{2 \pi \beta_{1}(x-\mu) / \alpha\right\}}{\alpha B\left(\beta_{1}, \beta_{2}\right)(1+\exp \{2 \pi(x-\mu) / \alpha\})^{\beta_{1}+\beta_{2}}}, \quad x \in \mathbb{R},
$$

where the set of parameters is

$$
\alpha>0, \quad \beta_{1}>0, \quad \beta_{2}>0, \quad \text { and } \quad \mu \in \mathbb{R}
$$

(see Prentice (1975) and Barndorff-Nielsen et al. (1982)). The characteristic function of a random variable $X$ with $\mathrm{PDF}$ (43) is given by

$$
\mathrm{Ee}^{\mathrm{i} z X}=\frac{B\left(\beta_{1}+\mathrm{i} \alpha z / 2 \pi, \beta_{2}-\mathrm{i} \alpha z / 2 \pi\right)}{B\left(\beta_{1}, \beta_{2}\right)} \mathrm{e}^{\mathrm{i} z \mu}, \quad z \in \mathbb{R} .
$$

This distribution has semiheavy tails and is known to be self-decomposable (Barndorff-Nielsen et al. (1982)) and, hence, infinitely divisible. Due to this infinite divisibility of the $z$-distribution, the following generalisation can be suggested.

We will use a stationary OU-type process (11) with marginal generalised $z$-distribution $Z\left(\alpha, \beta_{1}, \beta_{2}, \delta, \mu\right)$. The characteristic function of $X(t), t \in \mathbb{R}_{+}$, is then of the form

$$
\mathrm{Ee}^{\mathrm{i} z X}=\left(\frac{B\left(\beta_{1}+\mathrm{i} \alpha z / 2 \pi, \beta_{2}-\mathrm{i} \alpha z / 2 \pi\right)}{B\left(\beta_{1}, \beta_{2}\right)}\right)^{2 \delta} \mathrm{e}^{\mathrm{i} z \mu}, \quad z \in \mathbb{R},
$$

where the set of parameters is

$$
\alpha>0, \quad \beta_{1}>0, \quad \beta_{2}>0, \quad \delta>0, \quad \text { and } \quad \mu \in \mathbb{R} .
$$

This distribution is self-decomposable and, hence, infinitely divisible, with the Lévy triplet $(a, 0, v)$, where

$$
a=\frac{\alpha \delta}{\pi} \int_{0}^{2 \pi / \alpha} \frac{\exp \left\{-\beta_{2} x\right\}-\exp \left\{-\beta_{1} x\right\}}{1-\mathrm{e}^{-x}} \mathrm{~d} x+\mu
$$


and

$$
\begin{gathered}
\text { where } \quad b(u)= \begin{cases}\frac{2 \delta \exp \left\{-2 \pi \beta_{2} u / \alpha\right\}}{u\left(1-\mathrm{e}^{-2 \pi u / \alpha}\right)}, & u>0, \\
\frac{2 \delta \exp \left\{2 \pi \beta_{1} u / \alpha\right\}}{|u|\left(1-\mathrm{e}^{2 \pi u / \alpha}\right)}, & u<0 .\end{cases}
\end{gathered}
$$

Thus, if the $X_{j}(t), j=1, \ldots, m$, are independent so that $X_{j}(t) \sim Z\left(\alpha, \beta_{1}, \beta_{2}, \delta_{j}, \mu_{j}\right), j=$ $1, \ldots, m$, then we have

$$
X_{1}(t)+\cdots+X_{m}(t) \sim Z\left(\alpha, \beta_{1}, \beta_{2}, \delta_{1}+\cdots+\delta_{n}, \mu_{1}+\cdots+\mu_{n}\right) .
$$

The BDLP $Z(t)$ in (12) has a Lévy triplet $(\tilde{a}, 0, \tilde{v})$, where

$$
\tilde{a}=\lambda \mu+\frac{\alpha \lambda \delta}{\pi} \int_{0}^{\infty} \frac{\exp \left\{-\beta_{2} x\right\}-\exp \left\{-\beta_{1} x\right\}}{1-\mathrm{e}^{-x}} \mathrm{~d} x-\lambda \int_{|x|>1} x \omega(x) \mathrm{d} x,
$$

with the density of $\tilde{v}$ being given from

$$
\tilde{v}(\mathrm{~d} u)=\lambda \omega(u) \mathrm{d} u
$$

where

$$
\omega(u)= \begin{cases}\frac{4 \pi \delta}{\alpha}\left(\beta_{2} \exp \left\{-\frac{2 \pi \beta_{2}}{\alpha} u\right\}\left(1-\mathrm{e}^{-2 \pi u / \alpha}\right)+\exp \left\{-\frac{2 \pi\left(\beta_{2}+1\right)}{\alpha} u\right\}\right) & \\ \times \frac{1}{\left(1-\mathrm{e}^{-2 \pi u / \alpha}\right)^{2}}, & u>0, \\ \frac{4 \pi \delta}{\alpha}\left(\beta_{1} \mathrm{e}^{2 \pi \beta_{1} u / \alpha}\left(1-\mathrm{e}^{2 \pi u / \alpha}\right)+\exp \left\{\frac{2 \pi\left(\beta_{1}+1\right)}{\alpha} u\right\}\right) & \\ \times \frac{1}{\left(1-\mathrm{e}^{2 \pi u / \alpha}\right)^{2}}, & u<0 .\end{cases}
$$

The correlation function of the stationary process with marginal density (43) is then

$$
r_{X}(t)=\mathrm{e}^{-\lambda|t|}, \quad t \in \mathbb{R} .
$$

The PDF of the generalised $z$-distribution $Z\left(\alpha, \beta_{1}, \beta_{2}, \delta, \mu\right)$ has semiheavy tails:

$$
\pi(x) \sim C_{ \pm}|x|^{\rho_{ \pm}} \mathrm{e}^{-\sigma_{ \pm}|x|}, \quad|x| \rightarrow \pm \infty,
$$

where

$$
\rho_{ \pm}=2 \delta-1, \quad \sigma_{+}=\frac{2 \pi \beta_{2}}{\alpha}, \quad \sigma_{-}=\frac{2 \pi \beta_{1}}{\alpha}, \quad \text { and } \quad C_{ \pm}=\left(\frac{2 \pi}{\alpha B\left(\beta_{1}, \beta_{2}\right)}\right)^{2 \delta} \frac{\mathrm{e}^{ \pm \mu \sigma_{ \pm}}}{\Gamma(2 \delta)} .
$$

Note that

$$
\begin{gathered}
\mathrm{E} X(t)=\frac{\alpha \delta}{\pi} \int_{0}^{\infty} \frac{\exp \left\{-\beta_{2} x\right\}-\exp \left\{-\beta_{1} x\right\}}{1-\mathrm{e}^{-x}} \mathrm{~d} x+\mu, \\
\operatorname{var} X(t)=\frac{2 \alpha^{2} \delta}{(2 \pi)^{2}} \int_{0}^{\infty} x \frac{\exp \left\{-\beta_{2} x\right\}+\exp \left\{-\beta_{1} x\right\}}{1-\mathrm{e}^{-x}} \mathrm{~d} x .
\end{gathered}
$$


In particular, the generalised $z$-distribution $Z\left(\alpha, \frac{1}{2}+\beta / 2 \pi, \frac{1}{2}-\beta / 2 \pi, \delta, \mu\right)=M(\alpha, \beta, \delta, \mu)$ is known as the Meixner distribution (Schoutens and Teugels (1998), Grigelionis (1999), Morales and Schoutens (2003)). The density function of a Meixner distribution is given by

$$
\pi(x)=\frac{(2 \cos (\beta / 2))^{2 \delta}}{2 \pi \alpha \Gamma(2 \delta)} \exp \left\{\frac{\beta}{\alpha}(x-\mu)\right\}\left|\Gamma\left(\delta+\mathrm{i} \frac{x-\mu}{\alpha}\right)\right|^{2}, \quad x \in \mathbb{R},
$$

where

$$
\alpha>0, \quad-\pi<\beta<\pi, \quad \delta>0, \quad \text { and } \quad \mu \in \mathbb{R} \text {. }
$$

Note that

$$
|\Gamma(x+\mathrm{i} y)|^{2} \sim \sqrt{2 \pi}|y|^{x-1 / 2} \mathrm{e}^{-\pi|y| / 2} \quad \text { as }|y| \rightarrow \infty .
$$

This distribution is infinitely divisible and self-decomposable with triplet $(a, 0, v)$, where

$$
a=\alpha \delta \tan \frac{\beta}{2}-2 \delta \int_{1}^{\infty} \frac{\sinh (\beta x / 2)}{\sinh (\pi x / 2)} \mathrm{d} x+\mu
$$

and

$$
v(\mathrm{~d} u)=\frac{\delta \mathrm{e}^{\beta u / \alpha}}{u \sinh (\pi u / \alpha)} \mathrm{d} u .
$$

The cumulant function is

$$
C\{z ; X(t)\}=\mathrm{i} \mu z+2 \delta \log \frac{\cos (\beta / 2)}{\cosh ((\alpha z-\mathrm{i} \beta) / 2)}, \quad z \in \mathbb{R} .
$$

In particular, the hyperbolic cosine distribution $Z\left(\alpha, \frac{1}{2}, \frac{1}{2}, 0, \mu\right)=M\left(\alpha, 0, \frac{1}{2}, \mu\right)$ has the PDF

$$
\pi(x)=\frac{1}{\alpha \cosh (\pi(x-\mu) / \alpha)}, \quad x \in \mathbb{R},
$$

and characteristic function

$$
\mathrm{Ee}^{\mathrm{i} z X(t)}=\mathrm{e}^{\mathrm{i} z \mu} \frac{1}{\cosh (\alpha z / 2)}, \quad z \in \mathbb{R},
$$

while the logistic distribution $Z(\alpha, 1,1,0, \mu)$ has the PDF

$$
\pi(x)=\frac{2 \pi \exp (\pi(x-\mu) / \alpha)}{\alpha(1+\cosh (\pi(x-\mu) / \alpha))}, \quad x \in \mathbb{R},
$$

and characteristic function

$$
\mathrm{Ee}^{\mathrm{i} z X(t)}=\mathrm{e}^{\mathrm{i} z \mu} \frac{\alpha z}{2 \sinh (\alpha z / 2)}, \quad z \in \mathbb{R} .
$$

Another example is the $z$-distribution $Z\left(2 \pi, k_{1} / 2, k_{2} / 2,0, \log k_{1} / k_{2}\right)$, which is the $\log F_{k_{1}, k_{2}}$ distribution, where $F_{k_{1}, k_{2}}$ is the Fisher distribution (Barndorff-Nielsen et al. (1982)). Note that the generalised $z$-distributions and generalised hyperbolic distributions form nonintersecting sets. However, we can show that some Meixner distributions and corresponding Lévy processes can be obtained by subordination, that is, by random time change in the Brownian motion (see, for instance, Morales and Schoutens (2003)). 
(C8) Consider a mother process of the form

$$
\begin{gathered}
\Lambda(t)=\exp \left\{X(t)-c_{X}\right\} \\
\text { with } \quad c_{X}=2 \delta\left(\log \Gamma\left(\beta_{1}+\frac{\alpha}{2 \pi}\right)+\log \Gamma\left(\beta_{2}-\frac{\alpha}{2 \pi}\right)-\log \frac{\Gamma\left(\beta_{1}\right)}{\Gamma\left(\beta_{2}\right)}\right)+\mu,
\end{gathered}
$$

where $X(t), t \in \mathbb{R}_{+}$, is a stationary $Z\left(\alpha, \beta_{1}, \beta_{2}, \delta, \mu\right)$ OU-type process with covariance function

$$
R_{X}(t)=(\operatorname{var} X(t)) \mathrm{e}^{-\lambda|t|}, \quad t \in \mathbb{R} .
$$

The logarithm of the moment generating function of $Z\left(\alpha, \beta_{1}, \beta_{2}, \delta, \mu\right)$ is

$$
\begin{aligned}
K\{\zeta ; X(t)\}=2 \delta & \left(\log \Gamma\left(\beta_{1}+\frac{\alpha \zeta}{2 \pi}\right)+\log \Gamma\left(\beta_{2}-\frac{\alpha \zeta}{2 \pi}\right)\right. \\
& \left.-\log \frac{\Gamma\left(\beta_{1}\right)}{\Gamma\left(\beta_{2}\right)}\right)+\mu \zeta, \quad \zeta \in\left(-\frac{2 \pi \beta_{2}}{\alpha}, \frac{2 \pi \beta_{1}}{\alpha}\right)
\end{aligned}
$$

Under condition (C8), we obtain the moment generating function

$$
M(\zeta)=\operatorname{E} \exp \left\{\zeta\left(X(t)-c_{X}\right)\right\}=\exp \left\{-c_{X} \zeta\right\} \mathrm{e}^{K\{\zeta ; X(t)\}}, \quad|\beta+\zeta|<\alpha,
$$

and the bivariate moment generating function

$$
\begin{aligned}
M\left(\zeta_{1}, \zeta_{2} ;\left(t_{1}-t_{2}\right)\right) & =\operatorname{Eexp}\left\{\zeta_{1}\left(X\left(t_{1}\right)-c_{X}\right)+\zeta_{2}\left(X\left(t_{2}\right)-c_{X}\right)\right\} \\
& =\exp \left\{-c_{X}\left(\zeta_{1}+\zeta_{2}\right)\right\} \operatorname{Eexp}\left\{\zeta_{1} X\left(t_{1}\right)+\zeta_{2} X\left(t_{2}\right)\right\},
\end{aligned}
$$

where $\operatorname{E} \exp \left\{\zeta_{1} X\left(t_{1}\right)+\zeta_{2} X\left(t_{2}\right)\right\}$ is given by (16) with Lévy measure $\tilde{v}$ having density (44). Thus, the correlation function of the mother process takes the form

$$
\rho(\tau)=\frac{M(1,1 ; \tau)-1}{M(2)-1},
$$

where $M(2)$ is given by $(45)$ and $M(1,1 ; \tau)$ is given by (46).

Theorem 7. Suppose that condition (C8) holds, and let $Q=\left\{q\right.$ : $0<2 \pi \beta_{1} / \alpha<q<$ $\left.2 \pi \beta_{2} / \alpha, \beta_{1}<\beta_{2}\right\}$. Then, for any

$$
b>\exp \left\{-2 c_{X}\right\} \exp \left\{2 \delta\left(\log \Gamma\left(\beta_{1}+\frac{2 \alpha}{2 \pi}\right)+\log \Gamma\left(\beta_{2}-\frac{2 \alpha}{2 \pi}\right)-\log \frac{\Gamma\left(\beta_{1}\right)}{\Gamma\left(\beta_{2}\right)}\right)+2 \mu\right\},
$$

the stochastic processes $A_{n}(t)$ defined by (2) for the mother process (23) converge in $L_{2}$ to the stochastic process $A(t)$ as $n \rightarrow \infty$ such that, if $A(1) \in L_{q}$ for $q \in Q$,

$$
\mathrm{E} A(t)^{q} \sim t^{T(q)+1}
$$

where the Rényi function is given by

$$
\begin{aligned}
T(q)= & q\left(1+\frac{2 \delta\left(\log \Gamma\left(\beta_{1}+\alpha / 2 \pi\right)+\log \Gamma\left(\beta_{2}-\alpha / 2 \pi\right)-\log \left(\Gamma\left(\beta_{1}\right) / \Gamma\left(\beta_{2}\right)\right)\right)}{\log b}\right) \\
& -\frac{2 \delta}{\log b}\left(\log \Gamma\left(\beta_{1}+\frac{q \alpha}{2 \pi}\right)+\log \Gamma\left(\beta_{2}-\frac{q \alpha}{2 \pi}\right)\right)+\frac{1}{\log b} 2 \delta \log \frac{\Gamma\left(\beta_{1}\right)}{\Gamma\left(\beta_{2}\right)}-1 .
\end{aligned}
$$

Moreover, $\operatorname{var} A(t) \geq \int_{0}^{t} \int_{0}^{t}(M(1,1 ; u-w)-1) \mathrm{d} u \mathrm{~d} w$, where $M$ is given by (46). 
Proof. Theorem 7 follows from Theorems 1 and 2 and Proposition 1.

We can construct log- $z$ scenarios for a more general class of finite superpositions of stationary OU-type processes of the form (21), where the $X_{j}(t), j=1, \ldots, m$, are independent stationary processes with marginals $X_{j}(t) \sim Z\left(\alpha, \beta_{1}, \beta_{2}, \delta_{j}, \mu_{j}\right), j=1, \ldots, m$, and parameters $\delta_{j}, \mu_{j}, j=1, \ldots, m$. Then $X_{\text {sup }}(t), t \in \mathbb{R}_{+}$, has the marginal distribution $Z\left(\alpha, \beta_{1}, \beta_{2}, \sum_{j=1}^{m} \delta_{j}, \sum_{j=1}^{m} \mu_{j}\right)$ and covariance function

$$
R_{\text {sup }}(t)=\left(\frac{2 \alpha^{2}}{(2 \pi)^{2}} \int_{0}^{\infty} x \frac{\exp \left\{-\beta_{2} x\right\}+\exp \left\{-\beta_{1} x\right\}}{1-\mathrm{e}^{-x}} \mathrm{~d} x\right) \sum_{j=1}^{m} \delta_{j} \exp \left\{-\lambda_{j}|t|\right\}, \quad t \in \mathbb{R} .
$$

The generalisation of Theorem 2 and Proposition 1 to this situation is straightforward and the statement of Theorem 7 can be reformulated for the process of superposition $X_{\text {sup }}$ with $\delta=\sum_{j=1}^{m} \delta_{j}, \mu=\sum_{j=1}^{m} \mu_{j}$, and $M_{\boldsymbol{\theta}}\left(\zeta_{1}, \zeta_{2} ;\left(t_{1}-t_{2}\right)\right)=\prod_{j=1}^{m} M_{\boldsymbol{\theta}_{j}}\left(\zeta_{1}, \zeta_{2} ;\left(t_{1}-t_{2}\right)\right)$, where $\boldsymbol{\theta}=\left(\alpha, \beta_{1}, \beta_{2}, \delta, \mu\right)$ and $\boldsymbol{\theta}_{j}=\left(\alpha, \beta_{1}, \beta_{2}, \delta_{j}, \mu_{j}\right)$, and $\lambda$ must be replaced by $\lambda_{j}$ in the expression for $M_{\boldsymbol{\theta}_{j}}\left(\zeta_{1}, \zeta_{2} ;\left(t_{1}-t_{2}\right)\right)$, (46).

In a similar manner, we can construct scenarios such as the log-reciprocal inverse Gaussian scenario based on the results of Barndorff-Nielsen and Koudou (1998), the log-tempered stable scenario based on the results of Barndorff-Nielsen and Shephard (2002), (2003), and the log-Euler gamma scenario based on Euler's gamma distribution (see Grigelionis (2003)). In principle, it is possible to obtain log-hyperbolic scenarios for which there exist exact forms of Lévy measures of the OU process and the BDLP Lévy process; however, some analytical work is still to be carried out. This will be done elsewhere.

\section{Proofs of Theorem 2 and Proposition 1}

\subsection{Proof of Theorem 2}

Without loss of generality, we treat the case in which $\lambda=1$. Let $b \in \mathbb{R}$ be the drift, let $\sigma^{2} \geq 0$ be the variance of the Gaussian part, and let $N(\mathrm{~d} s, \mathrm{~d} u)$ be the Poisson random measure for the jumps with compensation $\tilde{N}(\mathrm{~d} s, \mathrm{~d} u)$ of the Lévy-Itô decomposition of $Z$. Let $B(t)$ denote the standard Brownian motion. Then the process $X$ is the stationary solution of the stochastic differential equation

$$
\mathrm{d} X(t)=(-X(t)+b) \mathrm{d} t+\sigma \mathrm{d} B(t)+\int_{|x|<1} x \tilde{N}(t, \mathrm{~d} x)+\int_{|x| \geq 1} x N(t, \mathrm{~d} x)
$$

(see, for example, Applebaum (2004, pp. 108, 216)). We apply Itô's formula to $f(x):=\mathrm{e}^{q x}$ to obtain

$$
\begin{aligned}
\mathrm{e}^{q X(t)}-\mathrm{e}^{q X(0)}= & \int_{0}^{t} q \mathrm{e}^{q X(s-)}(-X(s-)+b) \mathrm{d} s+\int_{0}^{t} q \sigma^{2} \mathrm{e}^{q X(s-)} \mathrm{d} B(s) \\
& +\frac{1}{2} \int_{0}^{t} q^{2} \sigma^{2} \mathrm{e}^{q X(s-)} \mathrm{d} s+\int_{0}^{t} \int_{|x| \geq 1}\left(\mathrm{e}^{q x}-1\right) \mathrm{e}^{q X(s-)} N(\mathrm{~d} s, \mathrm{~d} x) \\
& +\int_{0}^{t} \int_{|x|<1}\left(\mathrm{e}^{q x}-1\right) \mathrm{e}^{q X(s-)} \tilde{N}(\mathrm{~d} s, \mathrm{~d} x) \\
& +\int_{0}^{t} \int_{|x|<1}\left(\mathrm{e}^{q x}-1-q x\right) \mathrm{e}^{q X(s-)} v(\mathrm{~d} x) \mathrm{d} s
\end{aligned}
$$


We rearrange the above expression to get the following semimartingale decomposition (Applebaum (2004, p. 252)):

$$
\begin{aligned}
\mathrm{e}^{q X(t)}- & \mathrm{e}^{q X(0)} \\
= & \int_{0}^{t}\left(\mathrm{e}^{q X(s-)} q \sigma^{2} \mathrm{~d} B(s)+\int_{x \in \mathbb{R}} \mathrm{e}^{q X(s-)}\left(\mathrm{e}^{q x}-1\right) \tilde{N}(\mathrm{~d} s, \mathrm{~d} x)\right) \\
& +\int_{0}^{t} \mathrm{e}^{q X(s-)}\left(-X(s-) q+b q+\frac{1}{2} \sigma^{2} q^{2}+\int_{|x| \geq 1}\left(\mathrm{e}^{q x}-1\right) v(\mathrm{~d} x)\right. \\
& \left.+\int_{|x|<1}\left(\mathrm{e}^{q x}-1-q x\right) v(\mathrm{~d} x)\right) \mathrm{d} s .
\end{aligned}
$$

For the martingale part, by the Burkhölder-Gundy inequality (see Revuz and Yor (1991, p. 151) for the continuous case, and Bichteler (2002, p. 213) for the general case with jumps) and the quadratic variation of stochastic integrals (Applebaum (2004, p. 230)), we have, for each $t_{0}>0$,

$$
\begin{aligned}
\mathrm{E} \max _{0 \leq t \leq t_{0}}\left|\int_{0}^{t}\left(\mathrm{e}^{q X(s-)} \sigma^{2} \mathrm{~d} B(s)+\int_{x \in \mathbb{R}} \mathrm{e}^{q X(s-)}\left(\mathrm{e}^{q x}-1\right) \tilde{N}(\mathrm{~d} s, \mathrm{~d} x)\right)\right| \\
\leq C \mathrm{E}\left|\int_{0}^{t_{0}} \mathrm{e}^{2 q X(s-)} \sigma^{4} \mathrm{~d} s+\int_{0}^{t_{0}} \int_{\mathbb{R}} \mathrm{e}^{2 q X(s-)}\left(\mathrm{e}^{q x}-1\right)^{2} N(\mathrm{~d} s, \mathrm{~d} x)\right|^{1 / 2} .
\end{aligned}
$$

For the drift part,

$$
\begin{gathered}
\mathrm{E} \max _{0 \leq t \leq t_{0}} \mid \int_{0}^{t} \mathrm{e}^{q X(s-)}\left(-X(s-) q+b q+\frac{1}{2} \sigma^{2} q^{2}+\int_{|x| \geq 1}\left(\mathrm{e}^{q x}-1\right) v(\mathrm{~d} x)\right. \\
\left.\quad+\int_{|x|<1}\left(\mathrm{e}^{q x}-1-q x\right) v(\mathrm{~d} x)\right) \mathrm{d} s \mid \\
\leq \int_{0}^{t_{0}} \mathrm{E}\left(\mathrm{e}^{q X(s-)}\left(|X(s-)| q+|b| q+\frac{1}{2} \sigma^{2} q^{2}+C_{1}+C_{2}\right)\right) \mathrm{d} s,
\end{gathered}
$$

where

$$
C_{1}:=\int_{|x| \geq 1}\left|\mathrm{e}^{q x}-1\right| v(\mathrm{~d} x), \quad C_{2}:=\int_{|x|<1}\left|\mathrm{e}^{q x}-1-q x\right| v(\mathrm{~d} x),
$$

which are both finite by our assumption of the measure $v$.

We note that the process $t \rightarrow X(t-)$ is stationary and that, for each fixed $t, \mathrm{E} g(X(t))=$ $\mathrm{E} g(X(t-))$ for every nonnegative continuous function $g(\cdot)$ by the quasi-left-continuity of the process $Z$. For the drift part, the right-hand side of (47) is then less than or equal to

$$
\left(\mathrm{Ee}^{q X(0)}|X(0)| q+\mathrm{Ee}^{q X(0)}\left(|b| q+\frac{1}{2} \sigma^{2} q^{2}+C_{1}+C_{2}\right)\right) t_{0} .
$$

For the martingale part, we observe that $\mathrm{E} \sqrt{Y} \leq \sqrt{\mathrm{E} Y}$ for every nonnegative random variable $Y$; thus, the martingale part is less than or equal to

$$
\left(\mathrm{E}\left|\int_{0}^{t_{0}} \mathrm{e}^{2 q X(s-)} \sigma^{4} \mathrm{~d} s+\int_{0}^{t_{0}} \int_{R} \mathrm{e}^{2 q X(s-)}\left(\mathrm{e}^{q x}-1\right)^{2} N(\mathrm{~d} s, \mathrm{~d} x)\right|\right)^{1 / 2} .
$$

The first integral in (48) is less than or equal to

$$
\text { (constant) } \mathrm{Ee}^{2 q X(0)} t_{0} .
$$


Since $s \rightarrow X(s-)$ is left continuous, by the compensation formula in Kyprianou (2006, Theorem 4.4), the second integral in (48) is less than or equal to

$$
\text { (constant) } \mathrm{E} \mathrm{e}^{2 q X(0)} t_{0}\left(q^{2} \int_{|x| \leq 1} x^{2} v(\mathrm{~d} x)+\int_{|x| \geq 1}\left(\mathrm{e}^{q x}-1\right)^{2} v(\mathrm{~d} x)\right) .
$$

The assumption that $\int_{|x| \geq 1} g(x) v(\mathrm{~d} x)<\infty$, where $g(x)$ denotes any of the functions $\mathrm{e}^{2 q x}, \mathrm{e}^{q x}$, or $\mathrm{e}^{q x}|x|$, implies that $\mathrm{E} g(Z(t))<\infty$ for each $t$ (see, for example, Sato (1999, Theorem 25.3)). This again implies that $\mathrm{E} g(X(0))<\infty$ by Lemma 2.1 of Barndorff-Nielsen (2001).

The above arguments entail that

$$
\sum_{n=0}^{\infty} c\left(q, b^{-n}\right)<(\text { constant }) \sum_{n=0}^{\infty} \frac{1}{b^{n / 2}}<\infty,
$$

which proves the theorem.

\subsection{Proof of Proposition 1}

The proof depends on the Laplace transform of the Wiener-Lévy integral (Applebaum (2004, pp. 213, 214)). Let, for each $t>0$,

$$
Y(t):=\int_{0}^{t} f_{t}(s) \mathrm{d} Z(s)
$$

where the process $Z$ has drift $b$, with $\sigma^{2}=0$ and Lévy measure $\tilde{v}$ satisfying the integrability condition $\int_{|x|>1}|x| \tilde{v}(\mathrm{~d} x)<\infty$. The following lemma is an adaption of Applebaum (2004, pp. 91, 94).

Lemma 1. Assume that $\tilde{v}$ of Proposition 1 satisfies the exponential integrability condition that, for some $\theta>0, \int_{|x|>1} \mathrm{e}^{\theta x} \tilde{v}(\mathrm{~d} x)<\infty$. Then, for each $t>0$ and each $f_{t}(s)$ which is positive, increasing in $s$, and such that $f_{t}(s) \leq 1$,

$$
\begin{aligned}
\mathrm{Ee}^{\theta Y(t)}= & \exp \left\{\theta b \int_{0}^{t} f_{t}(s) \mathrm{d} s\right\} \\
& \times \exp \left\{\int_{0}^{t} \int_{\mathbb{R}}\left(\exp \left\{\theta f_{t}(s) x\right\}-1-\theta f_{t}(s) x \mathbf{1}_{[-1,1]}(x)\right) \tilde{v}(\mathrm{~d} x) \mathrm{d} s\right\} .
\end{aligned}
$$

Proof. The case in which $f_{t}(s) \equiv 1$ is given in Applebaum (2004, pp. 91, 94), written in the Laplace transform. The general case of positive continuous $f_{t}(s)$ with $f_{t}(s) \leq 1$ can be obtained by Riemann sum approximation.

Returning to the proof of Proposition 1 , we assume that $\lambda=1$. By the integral representation of $X$ we have

$$
X(t)=\mathrm{e}^{-t} X(0)+\int_{0}^{t} \mathrm{e}^{-(t-s)} \mathrm{d} Z(s),
$$

and the two parts of the right-hand side of (49) are independent (Applebaum (2004, p. 216)). Thus,

$$
\mathrm{E}\left(\mathrm{e}^{X(t)} \mathrm{e}^{X(0)}\right)=\mathrm{E} \exp \left\{\left(\mathrm{e}^{-t}+1\right) X(0)\right\} \mathrm{E}\left(\exp \left\{\int_{0}^{t} \mathrm{e}^{-(t-s)} \mathrm{d} Z(s)\right\}\right) .
$$

Now we fix a given $t>0$. The first expectation on the right-hand side of (50) is less than or equal to $\mathrm{E}^{2|X(0)|}$. We show that the second expectation has an exponential decay under the supposed 
conditions on $Z$ in Proposition 1. Applying Lemma 1 to $f_{t}(s):=f(s):=\mathrm{e}^{-(t-s)} \mathbf{1}_{\{0 \leq s \leq t-\delta\}}$ with $\theta=1$, where we choose $\delta$ such that $\mathrm{e}^{-\delta}<\beta$, we have

$$
\begin{aligned}
E \exp & \left\{\int_{0}^{t} \mathrm{e}^{-(t-s)} \mathrm{d} Z(s)\right\} \\
\leq & \mathrm{e}^{b(\beta-1)} \exp \left\{b\left(1-\mathrm{e}^{-t}\right)\right\} \exp \left\{\int_{0}^{t} \int_{|x| \leq 1} f^{2}(s) x^{2} \tilde{v}(\mathrm{~d} x) \mathrm{d} s\right\} \\
& \times \exp \left\{\int_{0}^{t} \int_{|x|>1}\left(\mathrm{e}^{f(s) x}-1\right) \tilde{v}(\mathrm{~d} x) \mathrm{d} s\right\} .
\end{aligned}
$$

For each $t>0, \exp \left\{b\left(1-\mathrm{e}^{-t}\right)\right\} \leq \mathrm{e}^{2|b|}$ and $\exp \left\{\int_{0}^{t} \int_{|x| \leq 1} f^{2}(s) x^{2} \tilde{v}(\mathrm{~d} x) \mathrm{d} s\right\} \leq \mathrm{e}^{c / 2}, c=$ $\int_{|x| \leq 1} x^{2} \tilde{v}(\mathrm{~d} x)$. Meanwhile,

$$
\begin{aligned}
& \exp \left\{\int_{0}^{t} \int_{|x|>1}\left(\mathrm{e}^{f(s) x}-1\right) \tilde{v}(\mathrm{~d} x) \mathrm{d} s\right\} \\
& \quad \leq \exp \left\{\int_{0}^{t} \int_{|x|>1} \mathrm{e}^{f(s) x} \tilde{v}(\mathrm{~d} x) \mathrm{d} s\right\} \mathrm{e}^{-t c^{\prime}}, \quad c^{\prime}=\tilde{v}(|x|>1)
\end{aligned}
$$

By the assumption on the density of $v$,

$$
\left.\int_{|x|>1} \mathrm{e}^{f(s) x} \tilde{v}(\mathrm{~d} x) \leq(\text { constant }) \int_{|x|>1} \mathrm{e}^{-(\beta-f(s))|x|} \mathrm{d} x \leq \text { constant }\right) \frac{1}{\beta-f(s)} \mathrm{e}^{-(\beta-f(s))} .
$$

We write $g(s)=\beta-f(s)$. By the definition of $f(s)$ we see that

$$
\int_{0}^{t} \frac{\mathrm{e}^{-g(s)}}{g(s)} \mathrm{d} s \leq \int_{0}^{t} \frac{-g^{\prime}(s)}{g(s)} \mathrm{d} s=\log \frac{1}{g(t)}-\log \frac{1}{g(0)},
$$

which is bounded in $t$. Therefore, for each given $t>0$,

$$
\operatorname{Eexp}\left\{-\int_{0}^{t} \mathrm{e}^{-(t-s)} \mathrm{d} Z(s)\right\} \leq(\text { constant }) \mathrm{e}^{-t c^{\prime}}
$$

$c^{\prime}=\tilde{v}(|x|>1)$, as required.

\section{Acknowledgements}

This research was partially supported by the Australian Research Council grant DP0559807, the National Science Foundation grant DMS-0417676, the EPSRC grant RCMT119, and the Taiwan NSC grant 962115M002005MY3. Leonenko's research was partially supported by the Welsh Institute of Mathematics and Computational Sciences. The authors are grateful to the anonymous referee for many constructive comments and suggestions to improve the paper; in particular, the accuracy of the proofs of Theorem 2 and Proposition 1.

\section{References}

AnH, V. V., LaU, K.-S. And Yu Z.-G. (2001). Multifractal characterization of complete genomes. J. Phys. A 34, 7127-7139.

Applebaum, D. (2004). Lévy Processes and Stochastic Calculus (Camb. Studies Adv. Math. 93). Cambridge University Press. 
BACRy, E. AND Muzy, J. F. (2003). Log-infinitely divisible multifractal processes. Commun. Math. Phys. 236, 449-475.

BARndorff-Nielsen, O. E. (1998). Processes of normal inverse Gaussian type. Finance Stoch. 2, 41-68.

BARndorff-Nielsen, O. E. (2001). Superposition of Ornstein-Uhlenbeck type processes. Theory Prob. Appl. 45, $175-194$.

Barndorff-Nielsen, O. E. And LeOnenKo, N. N. (2005). Spectral properties of superpositions of Ornstein-Uhlenbeck type processes. Methodology Comput. Appl. Prob. 7, 335-352.

BarndorfF-Nielsen, O. E. AND Koudou, A. E. (1998). Trees with random conductivities and the (reciprocal) inverse Gaussian distribution. Adv. Appl. Prob. 30, 409-424.

Barndorff-Nielsen, O. E. And Shephard, N. (2001). Non-Gaussian Ornstein-Uhlenbeck-based models and some of their uses in financial economics. J. R. Statist. Soc. B 63, 167-241.

Barndorff-Nielsen, O. E. And Shephard, N. (2002). Normal modified stable processes. Theory Prob. Math. Statist. 65, $1-20$.

BARNDORFF-NiELSEN, O. E. AND SHEPHARD, N. (2003). Integrated OU processes and non-Gaussian OU-based stochastic volatility models. Scand. J. Statist. 30, 277-295.

BarndorfF-Nielsen, O. E. ANd Shmigel, Yu. (2004). Spatio-temporal modeling based on Lévy processes, and its applications to turbulence. Uspekhi Mat. Nauk 59, 63-90 (in Russian). English translation: Russian Math. Surveys 59, 65-90.

Barndorff-Nielsen, O. E., Kent, J. And Sorenson, M. (1982). Normal variance-mean mixtures and $z$ distributions. Internat. Statist. Rev. 50, 145-159.

Barral J. And Mandelbrot, B. (2002). Multiplicative products of cylindrical pulses. Prob. Theory Relat. Fields 124, 409-430.

Bertoin, J. (1996) Lévy Processes (Camb. Tracts Math. 121). Cambridge University Press.

Bichteler, K. (2002). Stochastic Integration with Jumps (Encyclopaedia Math. Appl. 89). Cambridge University Press.

Carr, P., Geman, H., Madan, D. and Yor, M. (2007). Self-decomposability and option pricing. Math. Finance 17, 31-57.

FAlCONER, K. (1997). Techniques in Fractal Geometry. John Wiley, Chichester.

Finlay, R. And Seneta, E. (2006). Stationary-increment Student and variance-gamma processes. J. Appl. Prob. 43, 441-453. (Correction: 43 (2006), 1207).

FRISCH, U. (1995). Turbulence. Cambridge University Press.

FRISCH, U. AND PARISI, G. (1985). On the singularity structure of fully developed turbulence. In Proc. Internat. Summer School Phys., North-Holland, Amsterdam, pp. 84-88.

Grigelionis, B. (1999). Processes of Meixner type. Liet. Mat. Rink. 39, 40-51.

Grigelionis, B. (2001). Generalized z-distributions and related stochastic processes. Liet. Mat. Rink. 41, $303-319$.

Grigelionis, B. (2003). On the self-decomposability of Euler's gamma function. Liet. Mat. Rink. 43, 359-370.

Gupta, V. K. AND Waymire, E. C. (1993). A statistical analysis of mesoscale rainfall as a random cascade. J. Appl. Meteorology 32, 251-267.

Halsey, T. et al. (1986). Fractal measures and their singularities: the characterization of strange sets. Phys. Rev. A 33, 1141-1151.

Harte, D. (2001). Multifractals. Chapman \& Hall, Boca Raton, FL.

Hentschel, H. and Procaccia, I. (1983). The infinite number of generalized dimensions of fractals and strange attractors. Physica D 80, 435-444.

Jaffard, S. (1999). The multifractal nature of Lévy processes. Prob. Theory Relat. Fields 114, $207-227$.

Kahane, J.-P. (1985). Sur le chaos multiplicatif. Ann. Sci. Math. Québec 9, 105-150.

Kahane, J.-P. (1987). Positive martingales and random measures. Chinese Ann. Math. 8, 1-12.

Kolmogorov, A. N. (1941). Local structure of turbulence in fluid for very large Reynolds numbers. Dokl. Acad. Sci. USSR 31, 538-540.

Kolmogorov, A. N. (1962). On refinement of previous hypotheses concerning the local structure in viscous incompressible fluid at high Reynolds number. J. Fluid Mech. 13, 82-85.

KyPRIANOU, A. E. (2006). Introductory Lectures on Fluctuations of Lévy Processes with Applications. Springer, Berlin.

LAU, K.-S. (1999). Iterated function systems with overlaps and multifractal structure. In Trends in Probability and Related Analysis, eds N. Kono and N.-R. Shieh, World Scientific, River Edge, NJ.

Madan, D., CARr, P. And Chang, E. (1998). The variance gamma process and option pricing. Europ. Finance Rev. 2, 79-105.

Mandelbrot, B. (1997). Fractals and Scaling in Finance. Springer, New York.

Mannersalo, P., Norros, I. AND Riedi, R. (2002). Multifractal products of stochastic processes: construction and some basic properties. Adv. Appl. Prob. 34, 888-903.

Molchan, M. (1996). Scaling exponents and multifractal dimensions for independent random cascades. Commun. Math. Physics 179, 681-702. 
Morales, M. And Schoutens, W. (2003). A risk model driven by Lévy processes. Appl. Stoch. Models Bus. Ind. 19, $147-167$.

MöRTERS, P. AND SHIEH, N.-R. (2002). Thin and thick points for branching measure on a Galton-Watson tree. Statist. Prob. Lett. 58, 13-22.

MöRTERS, P. AND SHIEH, N.-R. (2004). On multifractal spectrum of the branching measure on a Galton-Watson tree. J. Appl. Prob. 41, 1223-1229.

Mörters, P. AND SHIEH, N.-R. (2008). Multifractal analysis of branching measure on a Galton-Watson tree. In Proc. 3rd Internat. Congress of Chinese Mathematicians (AMS/IP Studies in Advanced Mathematics 42), eds K.-S. Lau et al., Hong Kong, pp. 655-662.

Novikov, E. A. (1994). Infinitely divisible distributions in turbulence. Phys. Rev. E 50, R3303-R3305.

Prentice, R. L. (1975). Discrimination among some parametric models. Biometrika 62, 607-614.

Revuz, D. AND Yor, M. (1991). Continuous Martingales and Brownian Motion. Springer, Berlin.

RiedI, R. (2003). Multifractal processes. In Theory and Applications of Long-Range Dependence, eds P. Doukhan et al., Birkhäuser, Boston, MA, pp. 625-716.

SAITO, Y. (1992). Log-gamma distribution model of intermittency in turbulence. J. Phys. Soc. Japan 61, $403-406$.

SAto, K. (1999). Lévy Processes and Infinitely Divisible Distributions. Cambridge University Press.

Sснмітт, F. G. (2003). A causal multifractal stochastic equation and its statistical properties. Europ. J. Phys. B 34, 85-98.

Schmitt, F. G. AND Marsan, D. (2001). Stochastic equation generating continuous multiplicative cascades. Europ. J. Phys. B 20, 3-6.

Schoutens, W. And Teugels, J. L. (1998). Lévy processes, polynomials and martingales. Special Issue in honor of Marcel F. Neuts. Commun. Statist. Stoch. Models 14, 335-349.

ShIEH, N.-R. AND TAYloR, S. J. (2002). Multifractal spectra of branching measure on a Galton-Watson tree. J. Appl. Prob. 39, 100-111.

SkorokнoD, A. V. (1991). Random Processes with Independent Increments. Kluwer, Dordrecht.

TAYLOR, S. J. (1995). Super Brownian motion is a fractal measure for which the multifractal formalism is invalid. Fractals 3, 737-746. 